\title{
Precise mass and radius of a transiting super-Earth planet orbiting the M dwarf TOI-1235: a planet in the radius gap?
}

\author{
P. Bluhm ${ }^{1}$, R. Luque ${ }^{2,3}$, N. Espinoza ${ }^{4}$, E. Pallé2,3, J. A. Caballero 5 , S. Dreizler ${ }^{7}$, J. H. Livingston ${ }^{8}$, S. Mathur $^{2,3}$,
} A. Quirrenbach ${ }^{1}$, S. Stock ${ }^{1}$, V. Van Eylen ${ }^{9}$, G. Nowak ${ }^{2,3}$, E. D. López ${ }^{10,11}$, Sz. Csizmadia ${ }^{12}$, M. R. Zapatero Osorio ${ }^{13}$, P. Schöfer ${ }^{7}$, J. Lillo-Box ${ }^{5}$, M. Oshagh" ${ }^{2,3}$, E. González-Álvarez ${ }^{13}$, P. J. Amado ${ }^{6}$, D. Barrado ${ }^{5}$, V. J. S. Béjar 2,3, B. Cale ${ }^{14}$, P. Chaturvedi ${ }^{15}$, C. Cifuentes ${ }^{5}$, W. D. Cochran ${ }^{16}$, K. A. Collins ${ }^{17}$, K. I. Collins ${ }^{14}$, M. Cortés-Contreras ${ }^{5}$, E. Díez Alonso ${ }^{18,19}$, M. El Mufti ${ }^{14,20}$, A. Ercolino ${ }^{21}$, M. Fridlund ${ }^{22,23}$, E. Gaidos ${ }^{24}$, R. A. García ${ }^{25,26}$, I. Georgieva ${ }^{23}$, L. González-Cuesta ${ }^{2,3}$, P. Guerra ${ }^{27}$, A. P. Hatzes ${ }^{15}$, Th. Henning ${ }^{28}$, E. Herrero ${ }^{29,30}$, D. Hidalgo ${ }^{2,3}$, G. Isopi ${ }^{21}$, S. V. Jeffers ${ }^{7}$, J. M. Jenkins ${ }^{31}$, E. L. N. Jensen ${ }^{32}$, P. Kábath ${ }^{33}$, A. Kaminski ${ }^{1}$, J. Kemmer ${ }^{1}$, J. Korth ${ }^{34}$, D. Kossakowski ${ }^{28}$, M. Kürster ${ }^{28}$, M. Lafarga ${ }^{29,30}$, F. Mallia ${ }^{21}$, D. Montes $^{35}$, J. C. Morales ${ }^{29,30}$, M. Morales-Calderón ${ }^{5}$, F. Murgas ${ }^{2,3}$, N. Narita ${ }^{2,36,37,38}$, V. M. Passegger ${ }^{39,40}$, S. Pedraz ${ }^{41}$, C. M. Persson ${ }^{23}$, P. Plavchan ${ }^{14}$, H. Rauer ${ }^{12,42,43}$, S. Redfield ${ }^{44}$, S. Reffert ${ }^{1}$, A. Reiners ${ }^{7}$, I. Ribas ${ }^{29,30}$, G. R. Ricker ${ }^{45}$, C. Rodríguez-López ${ }^{6}$, A. R. G. Santos ${ }^{46}$, S. Seager ${ }^{45,47,48}$, M. Schlecker ${ }^{28}$, A. Schweitzer ${ }^{39}$, Y. Shan ${ }^{7}$, M. G. Soto ${ }^{49}$, J. Subjak ${ }^{33}$, L. Tal-Orr ${ }^{50,7}$, T. Trifonov ${ }^{28}$, S. Vanaverbeke ${ }^{51,52,}$ R. Vanderspek ${ }^{45}$, J. Wittrock ${ }^{14}$, M. Zechmeister ${ }^{7}$, and F. Zohrabi ${ }^{53}$

(Affiliations can be found after the references)

Received 13 April 2020 / Accepted 16 June 2020

\begin{abstract}
We report the confirmation of a transiting planet around the bright weakly active M0.5 V star TOI-1235 (TYC 4384-1735-1, $V \approx 11.5 \mathrm{mag}$ ), whose transit signal was detected in the photometric time series of sectors 14, 20, and 21 of the TESS space mission. We confirm the planetary nature of the transit signal, which has a period of $3.44 \mathrm{~d}$, by using precise RV measurements with the CARMENES, HARPS-N, and iSHELL spectrographs, supplemented by high-resolution imaging and ground-based photometry. A comparison of the properties derived for TOI-1235 b with theoretical models reveals that the planet has a rocky composition, with a bulk density slightly higher than that of Earth. In particular, we measure a mass of $M_{\mathrm{p}}=5.9 \pm 0.6 M_{\oplus}$ and a radius of $R_{\mathrm{p}}=1.69 \pm 0.08 R_{\oplus}$, which together result in a density of $\rho_{\mathrm{p}}=6.7_{-1.1}^{+1.3} \mathrm{~g} \mathrm{~cm}^{-3}$. When compared with other well-characterized exoplanetary systems, the particular combination of planetary radius and mass places our discovery in the radius gap, which is a transition region between rocky planets and planets with significant atmospheric envelopes. A few examples of planets occupying the radius gap are known to date. While the exact location of the radius gap for $\mathrm{M}$ dwarfs is still a matter of debate, our results constrain it to be located at around $1.7 R_{\oplus}$ or larger at the insolation levels received by TOI-1235 b $\left(\sim 60 S_{\oplus}\right)$. This makes it an extremely interesting object for further studies of planet formation and atmospheric evolution.
\end{abstract}

Key words. techniques: photometric - techniques: radial velocities - stars: individual: TOI-1235 - stars: late-type

\section{Introduction}

Currently, over 4000 exoplanetary systems have been discovered orbiting stars other than the Sun ${ }^{1}$, with the majority of the planets having sizes between that of the Earth and Neptune (Batalha et al. 2013). Most of these systems were discovered by the Kepler mission (Borucki et al. 2010; Borucki 2016), which by design focused its transit survey on stars of spectral types $\mathrm{F}, \mathrm{G}$, and $\mathrm{K}$. In order to understand the processes involved in the formation and evolution of planets, it is useful to compare the variations in the outcomes in different environments, for instance, by considering planetary demographics in a range of host star contexts. No picture of exoplanet populations can be complete without a sizable and representative sample of planetary systems around $\mathrm{M}$ dwarfs, which are the most common type of stars in our Galaxy (Chabrier 2003; Henry et al. 2006).

1 https://exoplanetarchive.ipac.caltech.edu/, http://exoplanet.eu/
The occurrence rate of small planets orbiting $M$ dwarfs indeed appears to increase toward late spectral subtypes at all orbital periods (Bonfils et al. 2013; Dressing \& Charbonneau 2015; Mulders et al. 2015; Gaidos et al. 2016). In spite of this abundance, the number of exoplanets with M-star hosts whose radii and masses are precisely known is still small because these stars are intrinsically faint, and only the closest ones are well suited for detailed follow-up and characterization.

One of the most interesting features observed in the distribution of sizes of small $\left(R<4 R_{\oplus}\right)$ exoplanets has been the bimodal nature of this distribution, which is commonly referred to as the "radius gap". It separates planets with radii slightly smaller than that of Neptune $\left(2-4 R_{\oplus}\right)$ from those with radii slightly larger than Earth $\left(1-2 R_{\oplus}\right)$. While the former are believed to bear a significant contribution of water (Morbidelli 2018), the latter are thought to be predominantly rocky. Although it was theoretically predicted (e.g., Owen \& Wu 2013; Jin et al. 2014; López \& Fortney 2014; Chen \& Rogers 2016), the radius gap was observationally characterized only relatively recently 
(e.g., Fulton et al. 2017; Zeng et al. 2017; Van Eylen et al. 2018; Berger et al. 2018; Fulton \& Petigura 2018) owing to an improvement in the planetary radius determination through more accurate models and stellar radii. This was possible through new high-resolution stellar spectroscopy (Schweitzer et al. 2019), asteroseismology (García \& Ballot 2019), and precise parallactic distances from the Gaia mission (Gaia Collaboration 2018).

Two classes of models are currently accepted to explain this radius gap: photoevaporation models, which posit that planets that finally lie below the radius gap lost their atmospheres due to X-ray and ultraviolet radiation from the star (XUV; e.g., Owen \& Wu 2013, 2017; López \& Fortney 2013; Jin et al. 2014; Chen \& Rogers 2016), and core-powered mass-loss models, which also propose that close-in planets below the radius gap have lost their atmospheres, but conjecture that mass loss is powered by heat from the planetary core (Ginzburg et al. 2016, 2018; Gupta \& Schlichting 2019). These two mechanisms have different dependences on the stellar type of the host stars and the total irradiation that the planets receive (Wu 2019; Gupta \& Schlichting 2020), which means that the actual location of the radius gap can indeed change with these parameters. Because most of the existing studies are based on Kepler samples or subsamples, which are samples that are heavily focused on F, G and K-type stars, transiting exoplanetary systems around M-type stars have a huge potential to help constrain the most important mechanism(s) producing this bimodal distribution (see, e.g., Hirano et al. 2018). Measuring the planetary mass in turn allows us to gain some insight into the bulk composition of the exoplanets, which delivers a clearer picture of the underlying nature of the radius gap. The Transiting Exoplanet Survey Satellite (TESS; Ricker et al. 2015) has proven to be a prime instrument for detecting and characterizing small planets orbiting bright stellar hosts. Having completed its first year of monitoring, it has contributed to the detection and confirmation of more than 40 new transiting exoplanetary systems, many of which consist of small planets orbiting low-mass M stars (e.g., Luque et al. 2019; Crossfield et al. 2019; Günther et al. 2019; Astudillo-Defru et al. 2020; Cloutier et al. 2020a; Gilbert et al. 2020; Nowak et al. 2020). Here we report on a very interesting addition to this growing sample of TESS transiting exoplanet discoveries around $\mathrm{M}$ dwarfs: a transiting super-Earth that appears to be right in the radius gap for low-mass stars orbiting the early M dwarf TOI-1235 (see also the coordinated, but intentionally independent, announcement by Cloutier et al. 2020b).

The paper is organized as follows. Section 2 presents the TESS photometry we used, along with ground-based observations of the star, including high-resolution spectroscopy, lucky and speckle imaging, and photometric variability monitoring. Section 3 presents the stellar properties of the host star, newly derived and collected from the literature. In Sect. 4 we present our analysis of the available data to constrain the planetary properties of the system. In Sect. 5 we discuss our results, with an emphasis on the location of the planet in the mass-radius diagram and its composition, and, finally, Sect. 6 shows our conclusions.

\section{Data}

Radius and mass are key physical properties of a planet. Together, they inform the planetary density, bulk composition, internal structure, and ability to retain an atmosphere. The combination of a transit and radial velocity (RV) detection is the most straightforward way to measure both the planetary radius and
Table 1. TESS observations of TOI- 1235 .

\begin{tabular}{cccll}
\hline \hline Sector & Camera & CCD & Start date & End date \\
\hline 14 & 4 & 3 & 18 July 2019 & 15 August 2019 \\
20 & 2 & 1 & 24 December 2019 & 21 January 2020 \\
21 & 2 & 2 & 21 January 2020 & 18 February 2020 \\
\hline
\end{tabular}

mass. In this work, we used the CARMENES ${ }^{2}$, HARPS-N ${ }^{3}$, and iSHELL $^{4}$ high-resolution spectrographs for the RV follow-up (Sect. 2.2).

Moreover, given the intrinsic faintness of $\mathrm{M}$ dwarfs in general and the large photometric apertures of wide-field surveys such as TESS in particular, many light curves with transit candidates are susceptible to contamination by nearby sources. Blends with stars other than the target star are frequent, especially at low Galactic latitudes, while many stars are unresolved multiples. In some cases, other stars in the aperture mask are variable and bright enough to affect the photometric, and even $\mathrm{RV}$, measurements. A particularly difficult type of false positives are background-eclipsing binaries near the target star, which can mimic planet transits. High-resolution imaging follow-up is therefore needed to identify nearby potential contaminants, and ground-based photometric monitoring is helpful in discarding false positives, such as nearby eclipsing binaries. For this second follow-up stage, we used the lucky and speckle imagers AstraLux and NESSI and collected light curves complementary to those of TESS, either measured by us with $1 \mathrm{~m}$ class telescopes (LCOGT, TJO) or compiled from public data bases (Sects. 2.3 and 2.4).

\subsection{TESS photometry}

The goal of TESS is to search for planets transiting bright and nearby stars. It was designed to observe 26 sectors of $24 \times 96 \mathrm{deg}^{2}$ split into 13 partially overlapping sectors in the north and south ecliptic hemispheres, each of which observed for 27-28 d. The Mikulski Archive for Space Telescopes ${ }^{5}$ (MAST) stores the light curves of stars in the TESS Input Catalog (TIC; Stassun et al. 2018). About 200000 bright main-sequence F, G, $\mathrm{K}$, and M-type stars, small enough to enable the detection of transiting planets smaller than Neptune $\left(R \leq 4 R_{\oplus}\right)$, are observed with a 2 min cadence (cf. Stassun et al. 2018), while fainter stars ( $V \gtrsim 13 \mathrm{mag}$ ) or with earlier spectral types (and, thus, high masses and large radii) are observed with a $30 \mathrm{~min}$ cadence. TOI-1235 (TIC 103633434) was observed by TESS in 2 min short-cadence integrations in sectors 14,20 , and 21 during the TESS primary mission (see Table 1). The transiting-planet signature was detected when the Science Processing Operations Center (SPOC; Jenkins et al. 2016) processed the data from sector 14 and searched the light curve for transits with the transiting planet search module (Jenkins 2002; Jenkins et al. 2017). The transit signature passed all the diagnostic tests performed by the data validation module (Twicken et al. 2018), which also provided the initial limb-darkened transit model fit ( $\mathrm{Li}$ et al. 2019). After these steps, TOI-1235 was announced on 16 September

2 Calar Alto high-Resolution search for M dwarfs with Exoearths with Near-infrared and optical Echelle Spectrographs: http: //carmenes. caha.es

3 High Accuracy Radial velocity Planet Searcher for the Northern hemisphere: https: //plone. unige.ch/HARPS-N/

4 Immersion Grating Echelle Spectrograph: http://irtfweb.ifa. hawaii . edu/ ishell/

5 https://mast.stsci.edu, https://archive.stsci.edu/ 

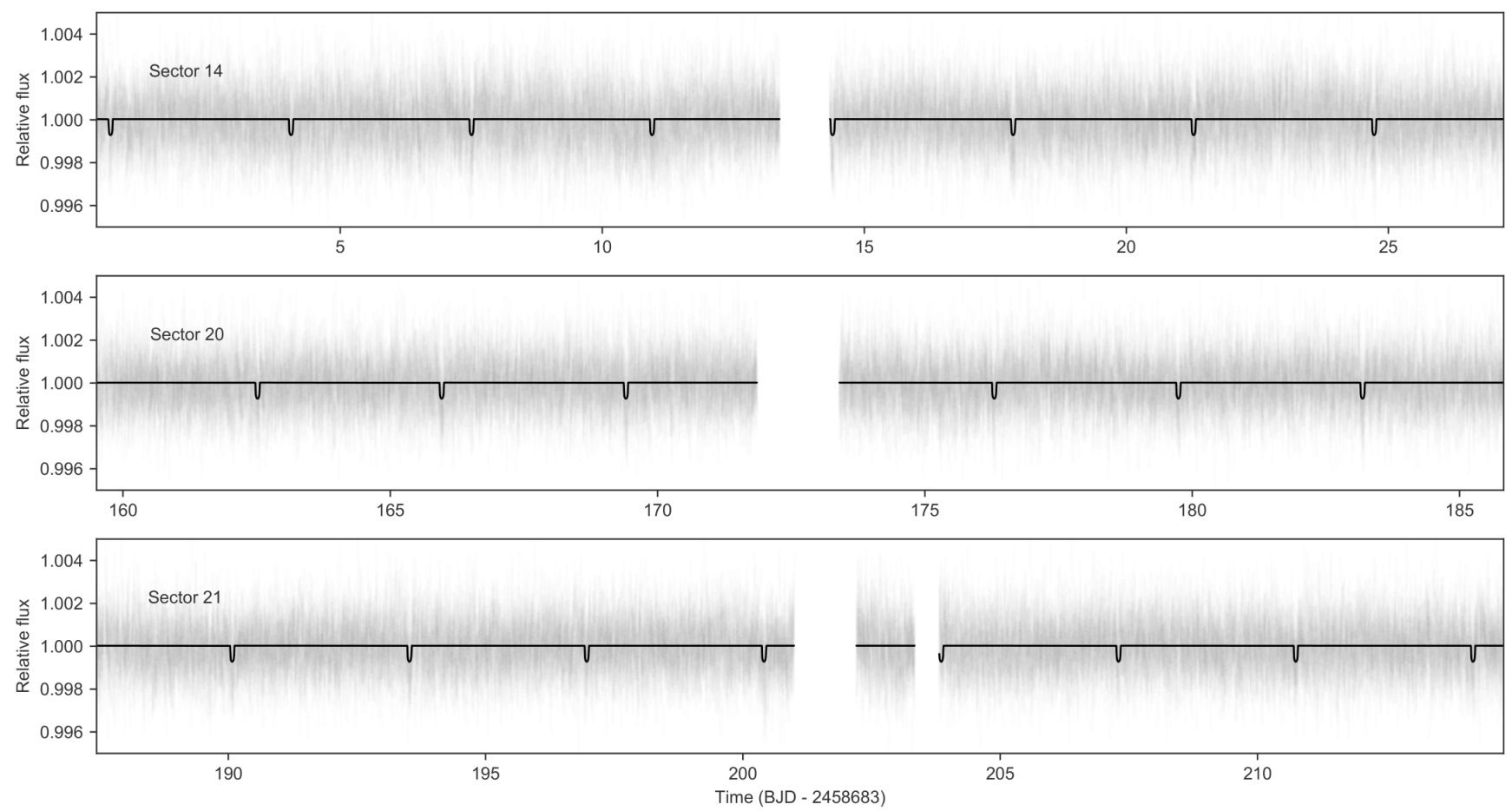

Sectors: 14,20 , and 21

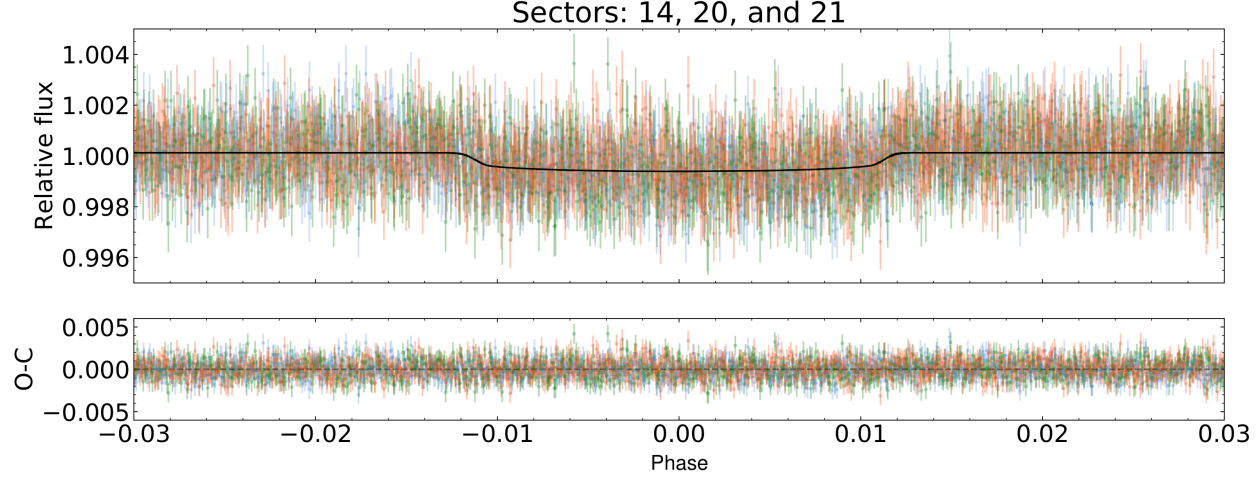

Fig. 1. Top panel: TESS transit photometry for the three sectors (gray points) with the best-fit juliet model (black line; see Sect. 4.4 for details on the modeling). Bottom panel: phase-folded transit light curve of TOI-1235 b. The three sectors (14, 20, and 21) are color-coded in red, green, and blue, respectively. The best-fit model is depicted by the black curve.

2019 as a TESS object of interest (TOI) thorugh the dedicated MIT TESS data alerts public website ${ }^{6}$. The transit signal had a period of $3.4431 \pm 0.0008 \mathrm{~d}$ and a depth of $0.91 \pm 0.08 \mathrm{mmag}$, corresponding to a planet radius of about $2 R_{\oplus}$, well in the super-Earth domain.

Soon after, we downloaded the corresponding light curve produced by the SPOC at the NASA Ames Research Center from MAST. SPOC provides simple aperture photometry (SAP) and photometry corrected for systematics (PDC, Smith et al. 2012; Stumpe et al. 2012, 2014), which is optimized for TESS transit searches. Figure 1 shows the PDC data for the three TESS sectors with the best-fit model (see Sect. 4.4 for details).

\subsection{High-resolution spectroscopy}

\subsubsection{CARMENES}

CARMENES (Quirrenbach et al. 2014, 2018) is a highresolution spectrograph mounted on the $3.5 \mathrm{~m}$ telescope at the Observatorio de Calar Alto in Almería, Spain. It splits

\footnotetext{
6 https://tess.mit.edu/toi-releases/
}

the incoming light into two channels, one that operates in the optical (VIS: $0.52-0.96 \mu \mathrm{m}, \mathcal{R}=94600$ ) and the other in the near-infrared (NIR: $0.96-1.71 \mu \mathrm{m}, \mathcal{R}=80400$ ). TOI-1235 was observed 40 times with CARMENES between 09 November 2019 and 18 February 2020, overlapping with the TESS sector 20 and 21 observations. We used the maximum exposure time of $1800 \mathrm{~s}$ and followed the standard data flow of the CARMENES guaranteed time observations. In particular, we reduced the VIS spectra with CARACAL (Zechmeister et al. 2014) and determined the corresponding RVs and spectral activity indices (see Sect. 4.3) with SERVAL (Zechmeister et al. 2018). We corrected the RV's for barycentric motion, instrumental drift, secular acceleration, and nightly zero-points (see Kaminski et al. 2018, Tal-Or et al. 2019, and, especially, Trifonov et al. 2020 for details). For exposure times shorter than $1800 \mathrm{~s}$, the CARMENES standard integrations are automatically limited by signal-to-noise ( $\mathrm{S} / \mathrm{N}$ ) ratio to 150 by an exposuremeter per channel that collects the light of the zeroth order of the respective échelle grating during the exposure (Caballero et al. 2016). However, the median S/N of our CARMENES VIS spectra was slightly lower $(\sim 97)$. Correspondingly, the weighted 
root-mean-square (wrms) and median uncertainty $(\hat{\sigma})$ of the CARMENES VIS data were 3.7 and $1.9 \mathrm{~m} \mathrm{~s}^{-1}$, respectively. These RVs and their uncertainties are listed in the top part of Table A.2.

As expected from the results presented by Bauer et al. (2020), the RV precision of the CARMENES NIR observations of TOI-1235 was lower than that of the VIS observations: wrms $=9.1$ and $\hat{\sigma}=7.4 \mathrm{~m} \mathrm{~s}^{-1}$. The expected RV amplitude of the planet of about $3-4 \mathrm{~m} \mathrm{~s}^{-1}$ was lower than the data radial precision of the CARMENES NIR data. The RVs, CRX, and dLW of CARMENES NIR spectra are displayed in the top part of Table A.3.

\subsubsection{HARPS-N}

HARPS-N (Cosentino et al. 2012) is a high-resolution spectrograph mounted on the Italian $3.58 \mathrm{~m}$ Telescopio Nazionale Galileo at the Observatorio del Roque de los Muchachos, La Palma, Spain. HARPS-N covers the optical wavelength regime between 0.38 and $0.69 \mu \mathrm{m}$ with a spectral resolution of $\mathcal{R}=115000$. The precision and stability of HARPS-N is comparable to its sister instrument HARPS on the ESO $3.6 \mathrm{~m}$ telescope and therefore to CARMENES (Trifonov et al. 2018; Perger et al. 2019). TOI-1235 was observed 21 times between 14 January 2020 and 26 February 2020 with HARPS-N ${ }^{7}$, also overlapping with TESS sectors 20 and 21 . Just as with the CARMENES data, we determined the RVs and $\mathrm{H} \alpha$ spectral activity index with SERVAL. They are listed in the bottom part of Table A.2. The typical S/N per exposure was 100 , while the wrms and $\hat{\sigma}$ of the HARPS-N data were 4.5 and $1.0 \mathrm{~m} \mathrm{~s}^{-1}$, respectively.

\subsection{3. iSHELL}

We obtained 49 spectra during five nights for TOI 1235 spanning 26 days in January-February 2020 with iSHELL mounted on the 3.2 m NASA Infrared Telescope Facility (IRTF) on Maunakea, Hawaii (Rayner et al. 2016). We used the silicon immersion grating optimized for the $K$ band with the 0.375 arcsec slit, which resulted in a spectral resolution of 80000 . The spectra were wavelength calibrated with a methane isotopolog gas cell in the calibration unit. The exposure times were $300 \mathrm{~s}$, repeated 9-11 times within a night to reach a cumulative photon $\mathrm{S} / \mathrm{N}$ per spectral pixel at about $2.4 \mu \mathrm{m}$ (at the approximate center of the blaze for the middle order) varying from 77 to 98 to achieve a per-night precision of 4-11 $\mathrm{m} \mathrm{s}^{-1}$. Spectra were reduced and RVs extracted using the methods outlined by Cale et al. (2019). The resulting wrms and $\hat{\sigma}$ of the iSHELL data were 7.2 and $6.1 \mathrm{~m} \mathrm{~s}^{-1}$, slightly better than the CARMENES NIR data, but still twice higher than the expected planet semiamplitude. The RVs are displayed in the bottom part of Table A.3.

\subsection{High-resolution imaging}

\subsubsection{AstraLux}

We observed TOI-1235 with the high spatial resolution camera and lucky imager AstraLux (Hormuth et al. 2008) on the $2.2 \mathrm{~m}$ telescope at the Observatorio de Calar Alto in Almería, Spain. The observations were carried out in the $z^{\prime}$ band on 30 October 2019 under good weather conditions with a mean seeing of 1.0 arcsec. We obtained 96000 frames of $10 \mathrm{~ms}$ in a $6.0 \times 6.0 \operatorname{arcsec}^{2}$ window. With the observatory pipeline, we

7 HARPS-N data: 15 RVs were obtained from the Spanish CAT19A162 program (PI: Nowak) and 6 RVs from ITP 19-1 program (PI: Pallé). selected the $5 \%$ frames with the highest Strehl ratio (Strehl 1902), aligned them, and stacked them for a final high-spatial resolution image.

\subsubsection{NESSI}

On 14 October of 2019, we observed TOI-1235 with the NASA Exoplanet Star and Speckle Imager (NESSI; Scott et al. 2018; Scott \& Howell 2018) on the $3.5 \mathrm{~m}$ WIYN telescope at the Kitt Peak National Observatory in Arizona, USA. We observed nearby point-source calibrator stars and reduced the data following Howell et al. (2011). The high-speed electron-multiplying CCDs of NESSI capture images at $25 \mathrm{~Hz}$ simultaneously in two bands centered at 562 and $832 \mathrm{~nm}$. Finally, we obtained two $4.6 \times 4.6 \operatorname{arcsec}^{2}$ reconstructed images, one for each passband.

\subsection{Ground-based photometry}

Additional photometric data for TOI-1235 were taken on 31 December 2019 with one of the $1 \mathrm{~m}$ telescopes of the Las Cumbres Observatory Global Telescope (LCOGT; Brown et al. 2013) Network at the McDonald Observatory in Texas, USA. We used the TESS Transit Finder, which is a customized version of the Tapir software package (Jensen 2013), to schedule a full transit observation. We used the $z s$ (short $z^{\prime}$ ) band and an aperture radius of 7.0 arcsec for the photometry extraction. A total of 358 photometric measurements were obtained with a cadence of $56 \mathrm{~s}$ and a median precision of $1.1 \mathrm{mmag}$ per point. The images were calibrated using the standard LCOGT Banzai pipeline (McCully et al. 2018), and the photometric data were extracted using the AstroImageJ software package (Collins et al. 2017).

We also observed a TOI-1235 transit on 29 March 2020 with the $0.8 \mathrm{~m}$ Telescopi Joan Oró (TJO) at the Observatori Astronòmic del Montsec in Lleida, Spain. We obtained a total of 221 images with the Johnson $R$ filter using the LAIA imager, a $4 \mathrm{k} \times 4 \mathrm{k}$ CCD with a field of view of 30 arcmin and a scale of 0.4 arcsec pixel ${ }^{-1}$. The observations were affected by poor weather conditions, and the photometry was extracted and analyzed with AstroImageJ. Although we did not use this photometry in the joint modeling due to the relatively poor photometric precision, of about $2 \mathrm{mmag}$, and an observational gap in the middle of the transit, it was still useful as an independent confirmation that the transit event indeed occurred on the target star, as the TJO photometry for all Gaia DR2 sources within 2.5 arcmin of the target ruled out the possibility that the TESS transit signal was produced by any of these stars being short-period eclipsing binary contaminants.

Finally, we searched for public time-series data of wide-area photometric surveys and databases exactly as in Díez Alonso et al. (2019). In particular, we retrieved light curves from the AllSky Automated Survey for SuperNovae (ASAS-SN; Shappee et al. 2014; Kochanek et al. 2017) in the $g^{\prime}$ and $V$ bands, and the Northern Sky Variability Survey (NSVS; Woźniak et al. 2004), and the Catalina Sky Survey (Drake et al. 2009) in white light. Table 2 summarizes the three public data sets, including the standard deviation of the magnitudes and mean of the magnitude errors. The Catalina data set is much noisier, sparser, and shorter than the others, therefore we did not use it in our analysis. In addition, we did not find data on TOI-1235 in the public archives of other photometric surveys, such as MEarth (Irwin et al. 2011), SuperWASP (Pollacco et al. 2006, including unpublished data), ASAS (Pojmański 1997), and HATNet (Bakos et al. 2004). Finally, TOI-1235 was not labeled as a variable star in the ATLAS survey (Heinze et al. 2018). 
Table 2. Descriptions of data from public ground-based surveys.

\begin{tabular}{llllccccc}
\hline \hline Survey & Band & Start date & End date & $N$ & $\begin{array}{c}\Delta t \\
(\mathrm{~d})\end{array}$ & $\begin{array}{c}\bar{m} \\
(\mathrm{mag})\end{array}$ & $\begin{array}{c}\sigma_{m} \\
(\mathrm{mag})\end{array}$ & $\begin{array}{c}\overline{\delta m} \\
(\mathrm{mag})\end{array}$ \\
\hline ASAS-SN & $g^{\prime}$ & 29 October 2017 & 24 March 2020 & $603^{(a)}$ & 877 & 12.255 & 0.026 & 0.010 \\
& $V$ & 28 January 2012 & 26 November 2018 & $713^{(b)}$ & 2494 & 11.572 & 0.018 & 0.009 \\
NSVS & Clear & 04 June 2018 & 20 May 2019 & 111 & 359 & 11.027 & 0.024 & 0.011 \\
Catalina $^{(b)}$ & Clear & 02 February 2006 & 18 April 2013 & 43 & 2632 & 10.761 & 0.089 & 0.050 \\
\hline
\end{tabular}

Notes. Number of collected data points. ${ }^{(a)}$ After discarding $20 g^{\prime}$ and $10 \mathrm{~V}$ dubious data points (with poor quality flags). ${ }^{(b)}$ Data set eventually not used.

\section{Stellar properties}

The star TOI-1235 (TYC 4384-1735-1) has been included in only a few proper-motion surveys (Høg et al. 2000; Lépine \& Shara 2005; Kirkpatrick et al. 2016) and catalogs of nearby M dwarfs that could host exoplanets (Lépine \& Gaidos 2011; Frith et al. 2013; Gaidos et al. 2014). As indicated by its Tycho-2 identifier, TOI- 1235 is a relatively nearby $(d \approx 39.6 \mathrm{pc})$ bright $(V \approx 11.5 \mathrm{mag})$ star. Lépine et al. (2013) and Gaidos et al. (2014) reported spectral types M0.5 V and M1.0 V and effective temperatures $T_{\text {eff }}$ of $3660 \mathrm{~K}$ and $4060 \mathrm{~K}$. Gaidos et al. (2014) also derived stellar radius $R_{\star}$ and bolometric luminosity $L_{\star}$, which are consistent with the determinations by Gaia Collaboration (2018), mass $M_{\star}$, and pseudo-equivalent width of the $\mathrm{H} \alpha$ line, $\mathrm{pEW}(\mathrm{H} \alpha)$.

We redetermined all stellar parameters for this early$\mathrm{M}$ dwarf. In particular, we measured $T_{\text {eff }}$, surface gravity $\log g$, and iron abundance $[\mathrm{Fe} / \mathrm{H}]$ from the stacked CARMENES VIS spectra by fitting them with a grid of PHOENIX-SESAM models as in Passegger et al. (2019), the rotational velocity $v \sin i$ with the cross-correlation method as in Reiners et al. (2018), and the stellar luminosity $L_{\star}$ by integrating the spectral energy distribution as in Cifuentes et al. (2020). To do this, we used photometric data in 17 passbands from the optical blue Tycho-2 $B_{\mathrm{T}}$ (Høg et al. 2000) to the mid-infrared AllWISE W4 (Cutri $\&$ et al. 2014), the Virtual Observatory Spectral energy distribution Analyzer (VOSA; Bayo et al. 2008), and the BT-Settl CIFIST theoretical models, which were used to extrapolate the spectral energy distribution at ranges bluer than $B_{\mathrm{T}}$ and redder than $W 4$. The full photometric data set including $u^{\prime}$ is made available by Cifuentes et al. (2020). The photospheric contributions to the total stellar flux of an $\mathrm{M} 0.5 \mathrm{~V}$ star in these ranges are $<0.5$ and $<0.004 \%$, which means that the $L_{\star}$ determination was model independent at the $>99.5 \%$ level at fixed metallicity. Next, we determined $R_{\star}$ through the Stefan-Boltzmann law, $L_{\star}=4 \pi R_{\star}^{2} \sigma T_{\mathrm{eff}}^{4}$, and $M_{\star}$ with the mass-radius relation derived from main-sequence eclipsing binaries by Schweitzer et al. (2019). All redetermined parameters $\left(T_{\text {eff }}, L_{\star}, R_{\star}\right.$, and $\left.M_{\star}\right)$ match the values published by Gaidos et al. (2014) and Gaia Collaboration (2018) within $1 \sigma$. Furthermore, we used the precise astrometric data of Gaia DR2, the absolute RV measured on the stacked CARMENES spectra as in Lafarga et al. (2020), and the prescription of Johnson \& Soderblom (1987) for measuring the Galactocentric space velocities $U V W$. Using this kinematic information with the BANYAN $\Sigma$ tool (Gagné et al. 2018), we classified TOI-1235 as a field star in the Galactic thin disk not associated with any young stellar kinematic group.

Finally, we determined key indicators of stellar activity. First, we measured the Mount Wilson $S$ index, $S_{\text {MwO }}$, with the Yabi data environment on the HARPS-N spectra (Hunter et al. 2012;
Borsa et al. 2015), from which we derived $\log R_{\mathrm{HK}}^{\prime}$ using the formulae of Astudillo-Defru et al. (2017) and $V-K_{s}=3.602 \pm$ 0.059 mag. Next, we measured $\mathrm{pEW}(\mathrm{H} \alpha)$ on the CARMENES stacked spectrum following Schöfer et al. (2019), which was in agreement within $2 \sigma$ to the $\mathrm{pEW}(\mathrm{H} \alpha)=+0.74 \pm 0.11 \AA$ measured by Gaidos et al. (2014) in April 2009. This means that the activity level of the star (as determined by $\mathrm{H} \alpha$ ) has not substantially changed for over a decade. These three indicators make TOI-1235 one of the least active stars for its spectral type (Wright et al. 2004; Astudillo-Defru et al. 2017; Boro Saikia et al. 2018). See Sect. 4.3 for a search for periodic signals in other spectroscopic activity indicators.

We also searched for soft X-ray and ultraviolet data of TOI-1235, but the star was not covered by any pointing (XMMNewton, Chandra, or EUVE), or was too faint and far from axis to be detected (ROSAT and GALEX). As an inactive member of the thin disk without further clear evidence to support a very young or very old age, TOI-1235 is likely between $0.6 \mathrm{Ga}$ (older than the Hyades) and $10 \mathrm{Ga}$ (younger than low-metallicity thick-disk stars).

Table 3 summarizes the stellar properties of TOI-1235. We provide the average values, their uncertainties, and corresponding reference.

\section{Analysis and results}

\subsection{Limits on photometric contamination}

The presence of an unresolved companion could have a strong effect on our results. This is particularly relevant for TESS photometry because of its large pixel size ( $21 \operatorname{arcsec})$. For comparison, the CARMENES and HARPS-N optical fiber apertures projected on the sky have sizes of only 1.5 arcsec and 1.0 arcsec, respectively. Even so, they are not immune to contamination from sub-arcsecond blends. Here, we place limits on the dilution factor and the presence of contaminant sources that can affect our photometric and RV measurements of TOI-1235. First, we verified that the sources in the selection apertures in the TESS pixel file (TPF) did not affect the depth of the transits significantly. The TPFs shown in Fig. 2 were created with tpfplotter ${ }^{8}$ (Aller et al. 2020). In particular, Gaia DR2 sources 2 and 3 in sectors 14 , source 4 in sector 20 , and source 8 in sector 21 all have $G$-band fluxes lower by $0.5 \%$ than that of TOI-1235 (Gaia and the TESS photometric bands are very similar). Similar results were found for the apertures of the ground-based surveys ASAS-SN and NSVS.

For subarcsecond separations, we used our lucky imaging AstraLux and speckle NESSI data sets described in Sect. 2.3

8 https://github.com/jlillo/tpfplotter 
Table 3. Stellar parameters of TOI-1235.

\begin{tabular}{|c|c|c|}
\hline Parameter & Value & Reference \\
\hline \multicolumn{3}{|c|}{ Name and identifiers } \\
\hline Name & TYC 4384-1735-1 & Нøg00 \\
\hline Karmn & J10088+692 & AF15 \\
\hline TOI & 1235 & ExoFOP-TESS \\
\hline TIC & 103633434 & Sta18 \\
\hline \multicolumn{3}{|c|}{ Coordinates and spectral type } \\
\hline$\alpha(\mathrm{J} 2000)$ & 10:08:52.38 & Gaia DR2 \\
\hline$\delta(\mathrm{J} 2000)$ & $+69: 16$ & Gaia DR2 \\
\hline Sp. type & $\mathrm{M} 0.5 \mathrm{~V}$ & Lep13 \\
\hline$G$ [mag] & $10.8492 \pm 0.0005$ & Gaia DR2 \\
\hline$T[\mathrm{mag}]$ & $9.9192 \pm 0.00$ & Sta19 \\
\hline$J[\mathrm{mag}]$ & $8.711 \pm 0.020$ & Skr06 \\
\hline \multicolumn{3}{|c|}{ Parallax and kinematics } \\
\hline$\varpi[\mathrm{mas}]$ & $25.202 \pm 0.030$ & Gaia DR2 \\
\hline$d[\mathrm{pc}]$ & $39.680 \pm 0.048$ & Gaia DR2 \\
\hline$\mu_{\alpha} \cos \delta\left[\mathrm{mas} \mathrm{a}^{-1}\right]$ & $+196.631 \pm 0.040$ & Gaia DR2 \\
\hline$\mu_{\delta}\left[\mathrm{mas} \mathrm{a}^{-1}\right]$ & $+17.369 \pm 0.047$ & Gaia DR2 \\
\hline$\gamma\left[\mathrm{km} \mathrm{s}^{-1}\right]$ & $-27.512 \pm 0.018$ & This work \\
\hline$U\left[\mathrm{~km} \mathrm{~s}^{-1}\right]$ & $+45.98 \pm 0.04$ & This work \\
\hline$V\left[\mathrm{~km} \mathrm{~s}^{-1}\right]$ & $-4.29 \pm 0.01$ & This work \\
\hline$W\left[\mathrm{~km} \mathrm{~s}^{-1}\right]$ & $+1.73 \pm 0.03$ & This work \\
\hline Gal. population & Thin disk & This work \\
\hline \multicolumn{3}{|c|}{ Photospheric parameters } \\
\hline$T_{\text {eff }}[\mathrm{K}]$ & $3997 \pm 51$ & This work \\
\hline $\log g$ & $4.64 \pm 0.04$ & This work \\
\hline$[\mathrm{Fe} / \mathrm{H}]$ & $+0.33 \pm 0.16$ & This work \\
\hline$v \sin i_{\star}\left[\mathrm{km} \mathrm{s}^{-1}\right]$ & $<2.0$ & This work \\
\hline \multicolumn{3}{|c|}{ Physical parameters } \\
\hline$L_{\star}\left[10^{-4} L_{\odot}\right]$ & $883 \pm 3$ & This work \\
\hline$\hat{M}_{\star}\left[M_{\odot}\right]$ & $0.630 \pm 0.024$ & This work \\
\hline$R_{\star}\left[R_{\odot}\right]$ & $0.619 \pm 0.019$ & This work \\
\hline \multicolumn{3}{|c|}{ Activity and age } \\
\hline $\mathrm{pEW}(\mathrm{H} \alpha)[\AA]$ & $+0.97 \pm 0.06$ & This work \\
\hline $\log R_{\mathrm{HK}}^{\prime}$ & $-4.728 \pm 0.015$ & This work \\
\hline & $1.005 \pm 0.029$ & This work \\
\hline Age $(\mathrm{Ga})$ & $0.6-10$ & This work \\
\hline
\end{tabular}

References. AF15: Alonso-Floriano et al. (2015); Gaia DR2: Gaia Collaboration (2018); Høg00: Høg et al. (2000); Lep13: Lépine et al. (2013); Schf19: Schöfer et al. (2019); Skr06: Skrutskie et al. (2006); Sta18: Stassun et al. (2018); Sta19: Stassun et al. (2019).

and illustrated by Fig. 3. We computed $5 \sigma$ contrast curves as described by Lillo-Box et al. (2012) with the astrasens package $^{9}$ for AstraLux, and as reported by Livingston et al. (2018) for NESSI. From both data sets, we confirmed the absence of any close companion 4-6 mag fainter than TOI-1235, and derived an upper limit to the contamination of around $2 \%$ between 0.15 arcsec and 1.5 arcsec (6.0-60 au if physically bound).

A further constraint came from the Gaia DR2 renormalized unit weight error (RUWE) value, which for TOI-1235 is 1.03, below the critical value of 1.40 that "indicates that a source is non-single or otherwise problematic for the astrometric solution" (Arenou et al. 2018; Lindegren et al. 2018). We also searched for wide common proper motion companions with similar Gaia

9 https://github.com/jlillo/astrasens
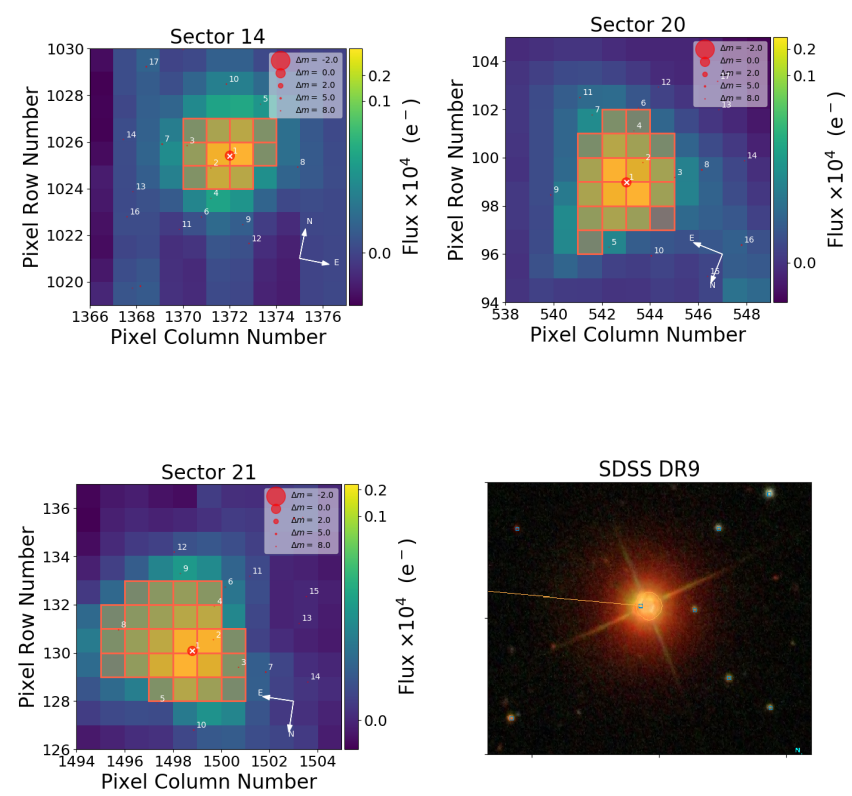

Fig. 2. Target pixel files of TOI-1235 in TESS sectors 14, 20, and 21. The electron counts are color-coded. The red bordered pixels are used in the SAP. The size of the red circles indicates the TESS magnitudes of all nearby stars and TOI-1235 (circle 1 is marked with a cross). Bottom right: false-color, $2 \times 2 \operatorname{arcmin}^{2}$ Sloan Digital Sky Survey DR9 image centered on TOI-1235 (north is up, east is left).

DR2 parallax, as in Montes et al. (2018), and found none within 30 arcmin of our star. Following these results, we conclude that TOI-1235 is a single star. We estimated the TESS and LCOGT dilution factors at $D=1.0$ with Eq. (2) in Espinoza et al. (2019), and fixed this value for all our model fits in the next sections.

\subsection{Stellar rotational period from photometric data}

The low activity levels of TOI-1235 probably imply a slow rotation and that there may not be enough spot coverage to measure a rotation period. Empirically, the measured limit on rotational velocity $\left(v \sin i<2 \mathrm{~km} \mathrm{~s}^{-1}\right)$ places a lower limit on $P_{\text {rot }} / \sin i>15.7 \mathrm{~d}$. Given the estimate of the stellar radius in Table 3, the short-period transiting planet around such a lowmass stars indicates a low obliquity (Winn et al. 2017), so that most probably, $\sin i \sim 1$ and therefore $P_{\text {rot }} \gtrsim 16 \mathrm{~d}$. On the other hand, from the $\log R_{\mathrm{HK}}^{\prime}-P_{\text {rot }}$ relation of Astudillo-Defru et al. (2017), TOI-1235 has a most likely $P_{\text {rot }}$ between $22 \mathrm{~d}$ and $38 \mathrm{~d}$. However, from Jeffers et al. (2018), the range of rotation periods that $\mathrm{M}$ dwarfs with $v \sin i<2 \mathrm{~km} \mathrm{~s}^{-1}$ can have is between $10 \mathrm{~d}$ and $150 \mathrm{~d}$.

To determine the actual rotational period of the star, we carried out different analyses of the available photometric data for TOI-1235. First, we employed the traditional periodogram analysis to search for significant peaks from the ASAS-SN $g^{\prime}$ - and $V$-band light curves. With the generalized Lomb-Scargle periodogram (GLS) of Zechmeister \& Kürster (2009), we obtained a peak at $48.63 \pm 0.08 \mathrm{~d}$ above the $10 \%$ false-alarm probability (FAP) threshold for the combined light curve after subtracting an independent zero-point from each band. We explored the time parameter space between $10 \mathrm{~d}$ and $1000 \mathrm{~d}$. Because the ASAS-SN light curves contained a significant number (15\%) of outlying data points because of flares and low S/Ns that might bias the previous GLS analysis, we repeated the GLS analysis after removing these deviant data from the two light curves in 

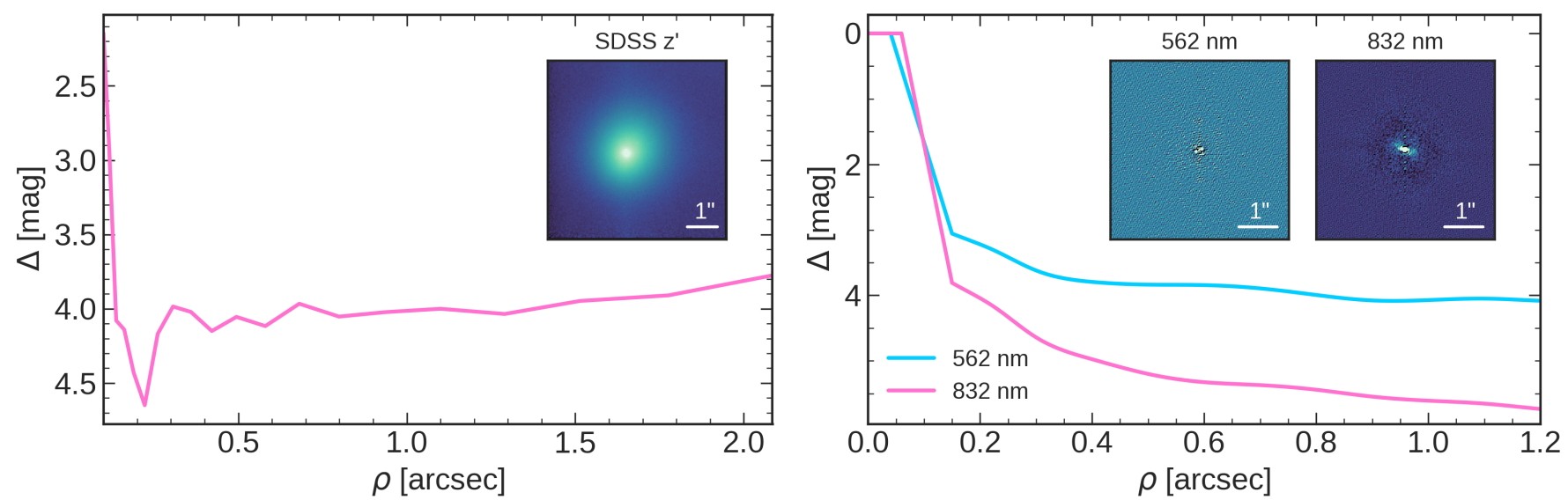

Fig. 3. Contrast curves $(5 \sigma)$ of TOI- 1235 from AstraLux (left) and NESSI (right) observations. Inset images are $6.0 \times 6.0 \operatorname{arcsec}^{2}$ stacked in $z^{\prime}$ band and $4.6 \times 4.6 \operatorname{arcsec}^{2}$ reconstructed at 562 and $832 \mathrm{~nm}$, respectively.

two steps: we first applied a $2 \sigma$ and then a $1 \sigma$ clipping algorithm. The new GLS periodogram of the resulting combined $g^{\prime}$ and $V$ data looked different to the one of the original ASAS-SN data, as there were no significant peaks in the studied parameter space. The highest peak near the $10 \%$ FAP level was located, but at a longer period of $136.9 \pm 1.4 \mathrm{~d}$. The marginal amplitude of the cleaned ASAS-SN $g^{\prime}$ - and $V$-band light curve folded in phase with this long period was only $1.4 \mathrm{mmag}$.

Next, we used a more sophisticated model and fit the ASASSN and NSVS photometry with a quasi-periodic Gaussian process (GP). In particular, we used the GP kernel introduced by Foreman-Mackey et al. (2017) of the form

$k_{i, j}(\tau)=\frac{B}{2+C} e^{-\tau / L}\left[\cos \left(\frac{2 \pi \tau}{P_{\text {rot }}}\right)+(1+C)\right]$,

where $\tau=\left|t_{i}-t_{j}\right|$ is the time lag, $B$ and $C$ define the amplitude of the GP, $L$ is a timescale for the amplitude-modulation of the GP, and $P_{\text {rot }}$ is the period of the quasi-periodic modulations. For the fit, we considered that each instrument and passband could have different values of $B$ and $C$, while $L$ and $P_{\text {rot }}$ were left as common parameters. We considered wide uninformative priors for $B, C$ (log-uniform between $10^{-3} \mathrm{ppm}$ and $\left.10^{8} \mathrm{ppm}\right), L(\log$ uniform between $10^{2} \mathrm{~d}$ and $10^{8} \mathrm{~d}$ ), $P_{\text {rot }}$ (uniform between $10 \mathrm{~d}$ and $300 \mathrm{~d}$ ), and instrumental jitter (log-uniform between $10 \mathrm{ppm}$ and $10^{6} \mathrm{ppm}$ ). The fit was performed using juliet (Espinoza et al. 2019, see the next section for a full description of the algorithm), and the resulting fit is presented in Fig. 4. The rotational period from the quasi-periodic GP analysis was found to be $P_{\text {rot }}=41.2_{-1.2}^{+1.1} \mathrm{~d}$, with an amplitude of about $10 \mathrm{mmag}$ during the time of highest stellar variability.

Finally, we took advantage of the TESS observations of TOI-1235 in three sectors spanning almost $210 \mathrm{~d}$. We analyzed the light curve described in Sect 2.1 and two light curves obtained from an optimized aperture (González-Cuesta et al., in prep.), in which we selected pixels with integrated flux above thresholds of 10 and $20 \mathrm{e}^{-} \mathrm{s}^{-1}$, respectively. We then corrected the light curves for outliers and jumps, filled the gaps, concatenated the three sectors following García et al. (2011, 2014a), and removed the transits to make sure that they did not bias the results. Last, we applied our rotation pipeline (Mathur et al. 2010; García et al. 2014b; Santos et al. 2019) with three different methods to search for a periodicity in the data: time-frequency analysis with wavelets (Torrence \& Compo 1998), autocorrelation function (McQuillan et al. 2014), a and composite spectrum
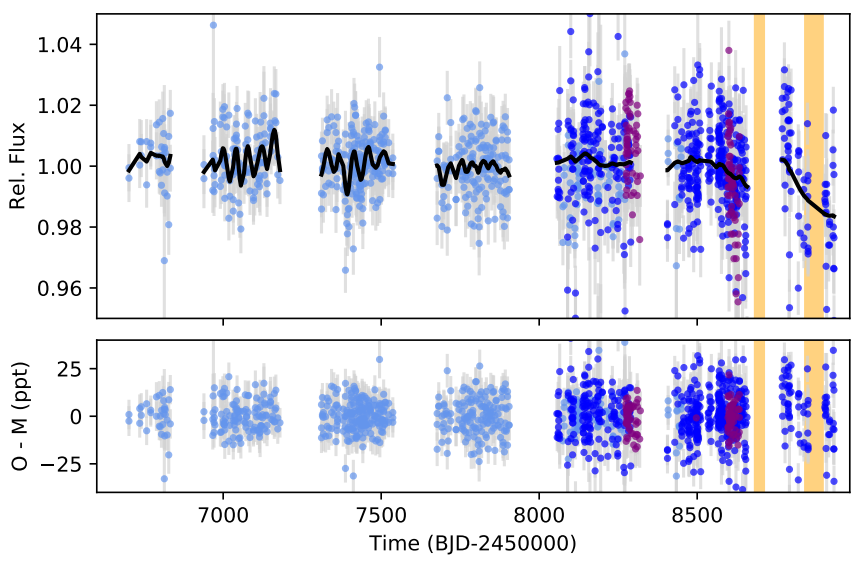

Fig. 4. ASAS-SN ( $V$ passband in light blue, $g^{\prime}$ passband in dark blue) and NSVS (purple) long-term photometric monitoring modeled with a quasi-periodic GP kernel defined as in Foreman-Mackey et al. (2017). The time span of the TESS observations is shown in gold. ASAS-SN and NSVS fluxes were computed from the original magnitudes and normalized with their respective median.

that is a combination of the two previous methods (Ceillier et al. 2017). While different combinations of methods and light curves generally yielded somewhat different periodicities, signals in the range 32-42 d were present in the time-frequency and composite spectrum analysis of the last two sectors. The significance of the peaks in the autocorrelation function and composite spectrum was slightly below the criteria of in Sect. 3.3 of Ceillier et al. (2017) for establishing a reliable period (i.e., height of the peaks in the autocorrelation functions and composite function greater than or equal than 0.30 and 0.15 , respectively). To summarize, the GLS periodogram of the raw ASAS-SN data (although with a low significance), the quasi-periodic GP modeling of the combined ASAS-SN and NSVS data, and the s-BGLS analysis of the spectroscopic data (see Sect. 4.3) all indicate a stellar origin of the $\sim 41.2 \mathrm{~d}$ photometric signal, which suggests that this value might be the true rotation period of TOI-1235.

\subsection{Signals in spectroscopic data}

We searched for periodic signals in the combined CARMENES VIS and HARPS-N RV data, which had the lowest median RV uncertainties, by computing GLS periodograms, as illustrated 


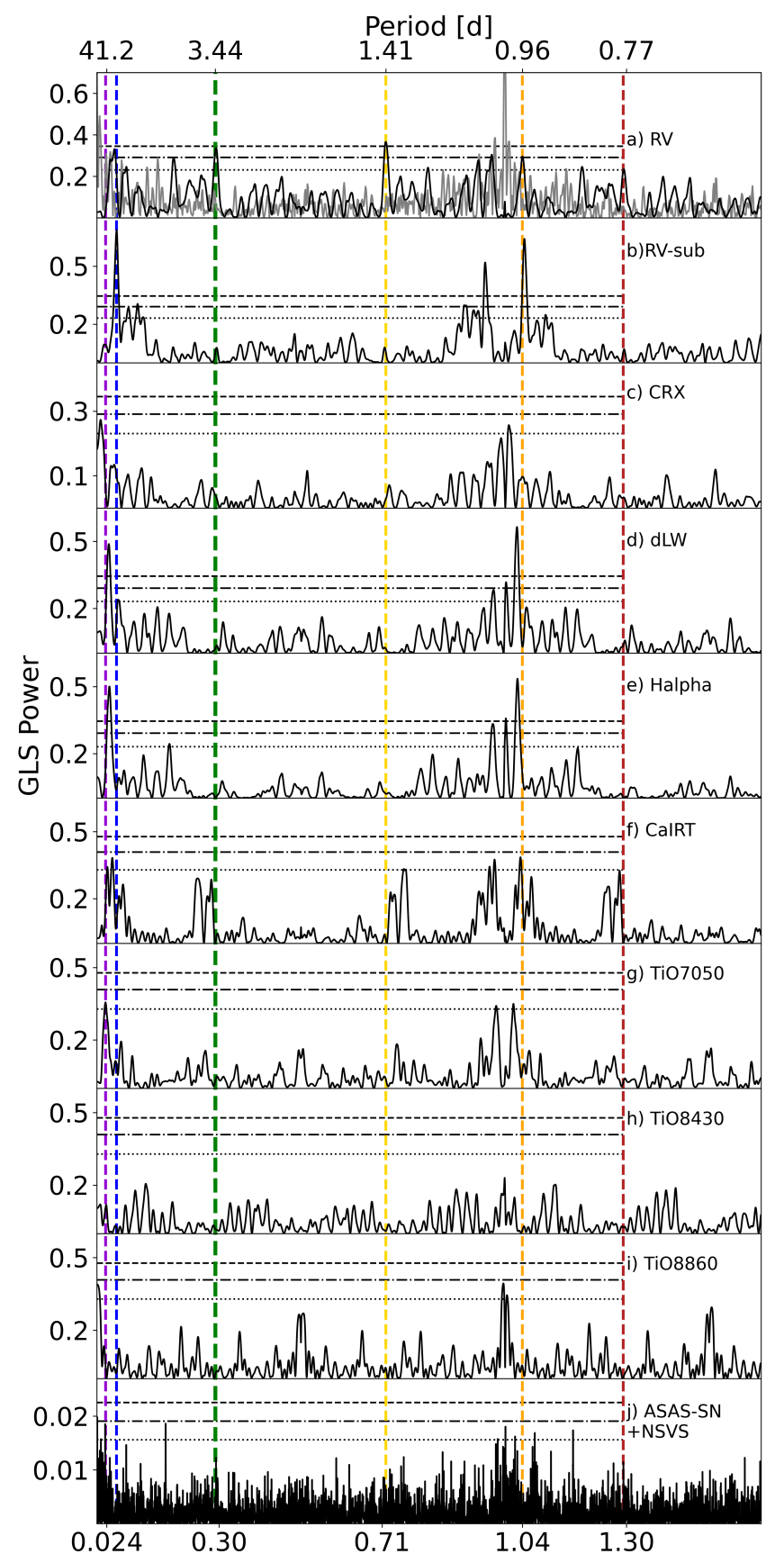

Fig. 5. GLS periodograms of: (a) combined RVs from CARMENES VIS and HARPS-N (black) and the respective spectral window function (gray), (b) RV residuals after subtracting the planet signal, $(c-e)$ combined CRX, dLW, and $\mathrm{H} \alpha$ index from CARMENES VIS and HARPS-N, and $(f-i)$ Ca IRT, TiO7050, TiO8430, and TiO8860 indices from CARMENES VIS alone, $(j)$ combined ASAS-SN ( $V$ and $g^{\prime}$ bands), and NSVS data photometry. In all panels the vertical dashed lines indicate the periods of $3.44 \mathrm{~d}$ (thick green, planet) and the aliases of the orbital period (yellow, orange, and red), $41.2 \mathrm{~d}$ (violet, $P_{\text {rot }}$ from the quasi-periodic GP analysis of the combined photometric data), $20.6 \mathrm{~d}$ (blue, $\sim P_{\text {rot }} / 2$ ). The horizontal lines mark the theoretical FAP levels of 0.1 (dashed), 1 (dash-dotted), and 10\% (dotted).

by Fig. 5. A signal corresponding to the transits in the TESS light curve was significantly detected in the RVs at $P_{\mathrm{b}}=3.44 \mathrm{~d}$ (FAP 1\%; panel a) and its aliases at $1.41 \mathrm{~d}, 0.96 \mathrm{~d}$, and $0.77 \mathrm{~d}$. However, we also found an additional signal at $P \approx 20.6 \mathrm{~d}$, at about half the most likely stellar rotation period. After
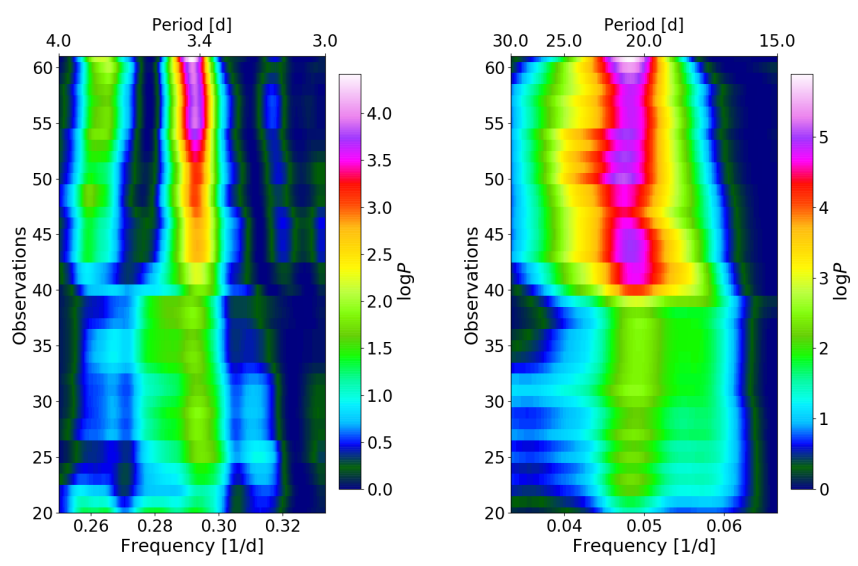

Fig. 6. Evolution of the s-BGLS periodogram of the CARMENES and HARPS-N RV data of TOI-1235 around the $3.44 \mathrm{~d}$ signal of the transiting planet (left) and around the $20.6 \mathrm{~d}$ activity signal after subtracting the planet signal (right). The number of data points included in the computation of the periodogram increases from bottom to top.

removing the planetary signal, the $20.6 \mathrm{~d}$ signal and its aliases still remained with an FAP $\gtrsim 0.1 \%$ (panel b).

To understand the origin of the $20.6 \mathrm{~d}$ signal, we searched for additional peaks in the periodograms of the activity indicators CRX, dLW, and $\mathrm{H} \alpha$ derived from the individual CARMENES and HARPS-N spectra (panels c-e), and Ca IRTa (panel f), and the titanium oxide indices that quantify the strengths of the TiO $\gamma, \epsilon$, and $\delta$ absorption band heads at 7050, 8430, and $8860 \AA$ (panels g-i), respectively, from the CARMENES spectra alone (Zechmeister et al. 2018; Schöfer et al. 2019). The activity indices and their uncertainties are listed in Table A.2. Except for daily aliases, the highest peaks in the $\mathrm{dLW}, \mathrm{H} \alpha, \mathrm{Ca}$ IRTa, and TiO7050 periodograms are at about $P \approx 32-47 \mathrm{~d}$, which adds further credence to $P_{\text {rot }} \approx 41.2 \mathrm{~d}$ as inferred in Sect. 4.2. All these indicators track different features in the stellar atmosphere, and our spectra cover only slightly more than two periods, therefore it is plausible that they do not yield exactly the same periods. We also detected the $20.6 \mathrm{~d}$ signal in the dLW series, which supports the notion that this signal is also related to stellar activity. As expected for an early-type M dwarf, the TiO8430 and TiO8860 indices showed no significant signals.

We used the stacked Bayesian generalized Lomb-Scargle periodogram (s-BGLS; Mortier et al. 2015) with the normalization of Mortier \& Collier Cameron (2017) to verify whether the $20.6 \mathrm{~d}$ signal was coherent over the whole observational time baseline of CARMENES VIS and HARPS-N. In Fig. 6 we display s-BGLS periodograms of the raw RV data around $3.44 \mathrm{~d}$, and of the RV data, after subtracting a sinusoid at the transiting planet period, around the $20.6 \mathrm{~d}$ signal.

This signal showed a first probability maximum after around 44 observations (BJD 2458 663) and thereafter decreased for some time. This incoherence is characteristic of a nonplanetary origin of the signal (Mortier \& Collier Cameron 2017). The s-BGLS of the $3.44 \mathrm{~d}$ signal, on the other hand, showed a monotonically increasing probability, as expected for a Keplerian signal.

Last, we measured the Pearson $r$, Student $t$, and Fisher $z$ correlation coefficients between the temporal series of RV and the activity indicators CRX, dLW, $\mathrm{H} \alpha$, Ca IRTa, TIO7050, and $S_{\text {MwO }}$, and we did not see any intrinsic correlation between RV and activity as in Gan et al. (2020). In particular, we determined absolute values of $r$ and $z$ below 0.006 and of $t$ above 0.7 , 
respectively, for all relations except for RV versus dLW, which was in any case weakly anticorrelated.

\subsection{Joint fit}

To obtain precise parameters of the TOI-1235 system, we performed a joint analysis of the TESS and LCOGT photometry and CARMENES VIS and HARPS-N RV data using juliet. The model that we selected for our RV joint fit analysis was one composed of a circular Keplerian orbit for the transiting planet plus a quasi-periodic GP that we used to model the $20.6 \mathrm{~d}$ signal observed in the RVs; we have discussed this in previous sections. However, we also computed models of a circular orbit, an eccentric orbit, a circular orbit plus a sinusoid, an eccentric orbit plus a sinusoid, an eccentric orbit plus a GP, and two circular orbits. The two best-fit models, judged by their log-evidences, were a two-planet model and a one-circular-planet model combined with a GP to fit the $20.6 \mathrm{~d}$ signal. The star-planet tidal locking and consequent circularization of the planet orbit following the theoretical predictions of Barnes (2017), for instance, support both models with eccentricity fixed to zero (see also Fig. 2 in Martínez-Rodríguez et al. 2019). However, the difference between their $\log$-evidence was $\Delta \ln \mathcal{Z}<2$, which made the two models indistinguishable if they were equally likely a priori. The two models gave almost identical constraints on the properties of the transiting exoplanet. The analyses of the activity indices and photometric data, however, gave a higher prior weight to the stellar activity model, and we therefore decided to use a GP, which is typically better at modeling stellar activity than a simple sinusoid, as our final model to account for the $20.6 \mathrm{~d}$ signal. In our analysis we used the exp-sine-squared kernel for the GP, which is a very common kernel to model stellar activity signatures in the literature (see, e.g., Nava et al. 2020, and references therein), and which is of the form

$k_{i, j}(\tau)=\sigma_{\mathrm{GP}}^{2} \exp \left(-\alpha \tau^{2}-\Gamma \sin ^{2}\left[\frac{\pi \tau}{P_{\text {rot }}}\right]\right)$.

For the transit modeling, juliet uses the batman package (Kreidberg 2015). To parameterize the limb-darkening effect in the TESS photometry, we employed the efficient, uninformative sampling scheme of Kipping (2013) and a quadratic law. We used a common set of limb-darkening coefficients across the three TESS sectors. In the LCOGT light-curve analysis, we instead used a linear law to parameterize the limb-darkening effect, as a more complex law was not warranted given the precision of the data, as explained by Espinoza \& Jordán (2016). We used the Espinoza (2018) parameterization to explore the full physically plausible parameter space for the planet-to-star radius ratio, $R_{\mathrm{p}} / R_{\star}$, and impact parameter, $b$. Finally, we used a white-noise-only fit for the TESS photometry, as an analysis using a GP on the photometry returned a log-evidence that was indistinguishable from the one of a white-noise model. For the LCOGT photometry, on the other hand, we used a linear model to detrend the data, with airmass and pixel position of the target as regressors. The selected priors for our joint fit are presented in Table A.1.

As illustrated by the posterior parameters of our joint fit presented in Table 4 and the resulting RV model presented in Fig. 7, the maximum a posteriori of the GP periodic component, $P_{\text {rot;GP,RV }}$, is about $20.9 \mathrm{~d}$, in agreement with the signal observed in the GLS periodogram of the RVs (Fig. 5). This is almost exactly half the period derived from the long-term photometric monitoring discussed in previous sections, which means that a rotating spotted stellar surface is the most plausible cause
Table 4. Posterior parameters of the juliet joint fit for TOI-1235 b.

\begin{tabular}{|c|c|}
\hline Parameter $^{(a)}$ & TOI-1235 b \\
\hline \multicolumn{2}{|c|}{ Stellar parameters } \\
\hline$\rho_{\star}\left(\mathrm{g} \mathrm{cm}^{-3}\right)$ & $3.74_{-0.31}^{+0.30}$ \\
\hline \multicolumn{2}{|c|}{ Planet parameters } \\
\hline$P(\mathrm{~d})$ & $3.444717_{-0.000042}^{+0.000040}$ \\
\hline$t_{0}(\mathrm{BJD})$ & $2458683.6155_{-0.0015}^{+0.0017}$ \\
\hline$a / R_{\star}$ & $13.29_{-0.38}^{+0.34}$ \\
\hline$p=R_{\mathrm{p}} / R_{\star}$ & $0.02508_{-0.00085}^{+0.000}$ \\
\hline$b=\left(a / R_{\star}\right) \cos i_{\mathrm{p}}$ & $0.25_{-0.14}^{+0.12}$ \\
\hline$i_{\mathrm{p}}(\operatorname{deg})$ & $88.90_{-0.57}^{+0.62}$ \\
\hline$r_{1}$ & $0.500_{-0.097}^{+0.081}$ \\
\hline$r_{2}$ & $0.02506_{-0.00085}^{+0.00083}$ \\
\hline$K\left(\mathrm{~m} \mathrm{~s}^{-1}\right)$ & $3.40_{-0.34}^{+0.35}$ \\
\hline \multicolumn{2}{|c|}{ Photometry parameters } \\
\hline$M_{\mathrm{TESS}, \mathrm{S} 14}\left(10^{-6}\right)$ & $-31.0_{-8.3}^{+8.5}$ \\
\hline$M_{\mathrm{TESS}, \mathrm{S} 20}\left(10^{-6}\right)$ & $-17.0_{-8.2}^{+8.3}$ \\
\hline$M_{\mathrm{TESS}, \mathrm{S} 21}\left(10^{-6}\right)$ & $-24.0_{-8.0}^{+8.0}$ \\
\hline$\sigma_{\mathrm{TESS}, \mathrm{S} 14}(\mathrm{ppm})$ & $1.9_{-1.6}^{+10.5}$ \\
\hline$\sigma_{\mathrm{TESS}, \mathrm{S} 20}(\mathrm{ppm})$ & $1.9_{-1.6}^{+8.2}$ \\
\hline$\sigma_{\mathrm{TESS}, \mathrm{S} 21}(\mathrm{ppm})$ & $1.5_{-1.3}^{+7.8}$ \\
\hline$q_{1, \mathrm{TESS}}$ & $0.42_{-0.25}^{+0.32}$ \\
\hline$q_{2, \mathrm{TESS}}$ & $0.31_{-0.20}^{+0.30}$ \\
\hline$M_{\mathrm{LCO}}\left(10^{-6}\right)$ & $-257_{-86}^{+84}$ \\
\hline$\sigma_{\mathrm{LCO}}(\mathrm{ppm})$ & $970_{-83}^{+82}$ \\
\hline$q_{1, \mathrm{LCO}}$ & $0.49_{-0.30}^{+0.30}$ \\
\hline$\theta_{0, \mathrm{LCO}}\left(10^{-6}\right)$ & $-10_{-11}^{+11}$ \\
\hline$\theta_{1, \mathrm{LCO}}\left(10^{-6}\right)$ & $-49_{-11}^{+11}$ \\
\hline \multicolumn{2}{|c|}{$R V$ parameters } \\
\hline$\gamma_{\text {CARMENES }}\left(\mathrm{m} \mathrm{s}^{-1}\right)$ & $-3.0_{-4.3}^{+4.6}$ \\
\hline$\sigma_{\text {CARMENES }}\left(\mathrm{m} \mathrm{s}^{-1}\right)$ & $0.17_{-0.14}^{+0.61}$ \\
\hline$\gamma_{\text {HARPS-N }}\left(\mathrm{m} \mathrm{s}^{-1}\right)$ & $3.8_{-4.2}^{+4.6}$ \\
\hline$\sigma_{\text {HARPS-N }}\left(\mathrm{m} \mathrm{s}^{-1}\right)$ & $1.29_{-0.37}^{+0.43}$ \\
\hline \multicolumn{2}{|c|}{ GP hyperparameters } \\
\hline$\sigma_{\mathrm{GP}, \mathrm{RV}}\left(\mathrm{m} \mathrm{s}^{-1}\right)$ & $12.3_{-6.3}^{+17.9}$ \\
\hline$\alpha_{\mathrm{GP}, \mathrm{RV}}\left(10^{-6} \mathrm{~d}^{-2}\right)$ & $74_{-50}^{+127}$ \\
\hline$\Gamma_{\mathrm{GP}, \mathrm{RV}}$ & $0.084_{-0.068}^{+0.251}$ \\
\hline$P_{\text {rot;GP,RV }}(\mathrm{d})$ & $20.93_{-0.52}^{+0.56}$ \\
\hline
\end{tabular}

Notes. ${ }^{(a)}$ Priors and descriptions for each parameter are in Table A.1. Error bars denote the $68 \%$ posterior credibility intervals.

of these variations. Consequently, we performed joint fits using the period observed in the photometry of $P_{\text {rot }}=41.2_{-1.5}^{+1.2} \mathrm{~d}$ as a prior, and the results were almost identical regarding the properties of the transiting planet to the ones presented in Table 4. Therefore our model is marginalized properly over the possible different scenarios on the stellar surface in terms of stellar activity. As shown in Fig. 8 and Table 4, we attained a $10 \sigma$ detection of the planetary RV semiamplitude.

We also performed two additional fits of the lower-precision CARMENES NIR and iSHELL RVs (Sects. 2.2.1 and 2.2.3). We set all the ephemeris priors to those found in the joint fit, 

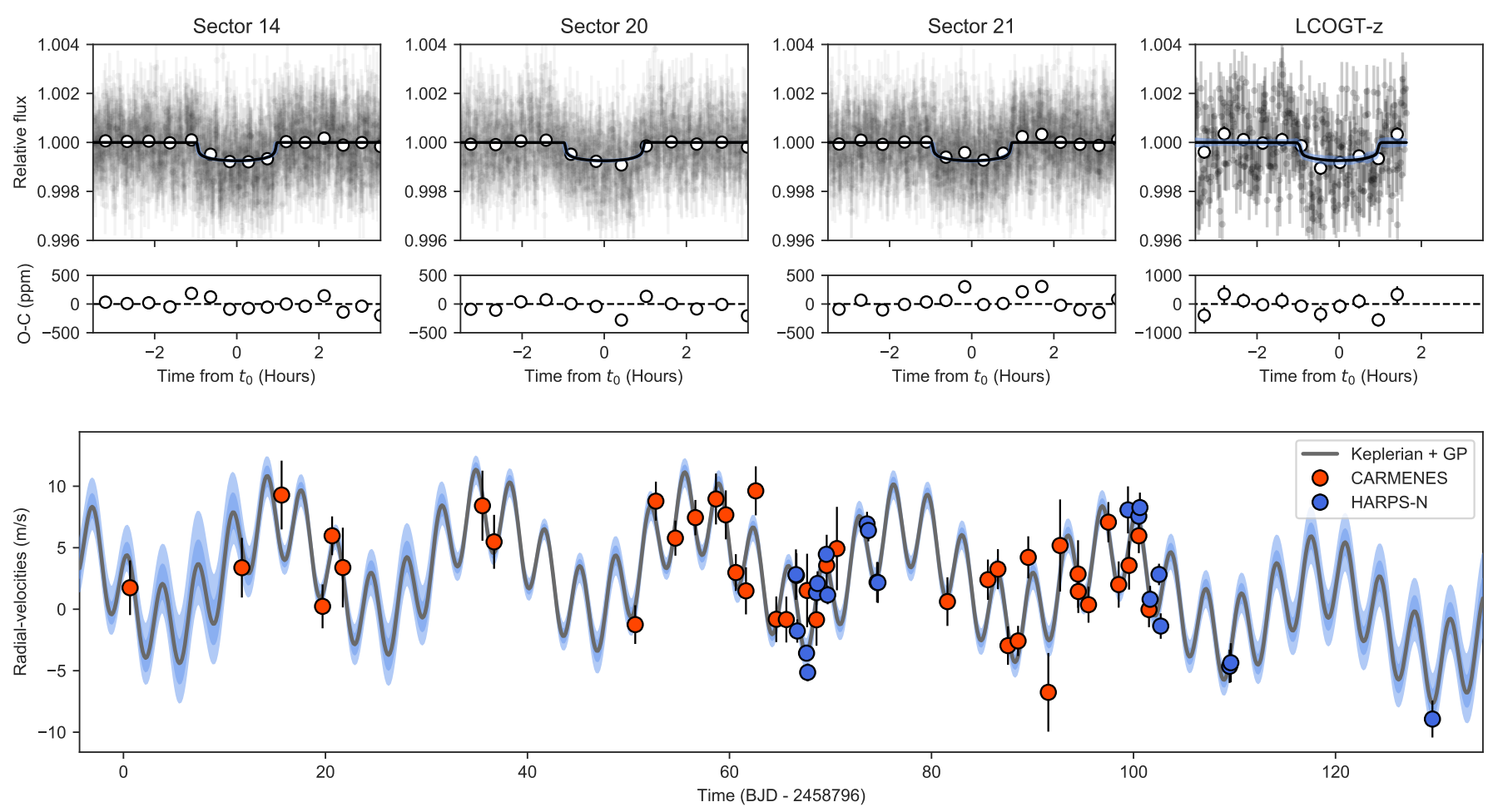

Fig. 7. Joint fit results. Top panels: phase-folded light curves of TESS, sectors 14, 20, and 21, and LCOGT, from left to right, and their residuals. White circles are binned data (shown only for reference; data used to fit the model were the unbinned points), black curves are the best-fit models, and blue areas are the $68 \%$ credibility bands. Bottom panel: CARMENES VIS (orange) and HARPS-N (blue) RVs. The gray curve is the median best-fit juliet model, and the light and dark blue areas are its 68and 95\% credibility bands.
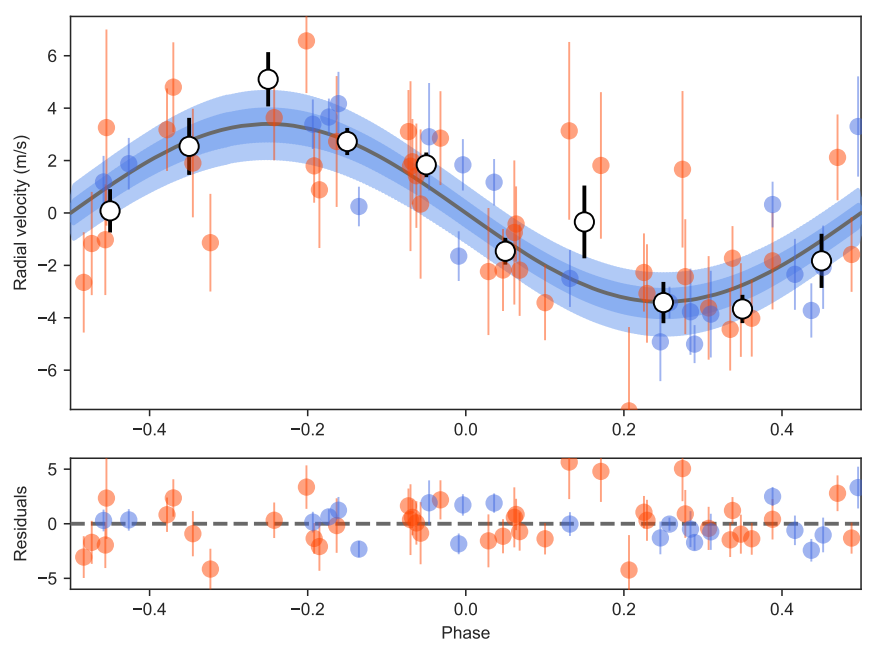

Fig. 8. Phase-folded RVs for TOI-1235 without the GP component. Orange circles are CARMENES VIS data, blue circles are HARPS-N data, white points are binned data for reference. The gray curve is the median best-fit juliet model, and the light and dark blue areas are its 68 and $95 \%$ credibility bands.

including the planet $P$ and $t_{0}$, with and without stellar rotation period and timescale of the GP. All other parameters were free to vary around the entire parameter space. The two models, with and without GP, were indistinguishable based on their logevidences $(\Delta \ln \mathcal{Z}<1)$. Interestingly, the GP amplitude in the first model was consistent with zero, which supports the nonplanetary origin of the $\sim 41.2 \mathrm{~d}$ period, as argued above. The new NIR RV analysis yielded a lower statistical precision in model parameter recovery than VIS RVs, but the new recovered planet-semiamplitude $K_{\mathrm{NIR}}=2.8 \pm 1.4 \mathrm{~m} \mathrm{~s}^{-1}$ was consistent within $1 \sigma$ with that listed in Table 4 . In addition, the NIR RVs, taken at independent wavelengths and in the case of iSHELL with a different facility, help validate the system and instrument performance.

To summarize, the TOI-1235 system consists of a relatively weakly active $\mathrm{M}$ dwarf with at least one super-Earth-like planet, namely TOI- $1235 \mathrm{~b}$ (see Table 5), with a mass of $M_{\mathrm{p}}=5.9_{-0.6}^{+0.6} M_{\oplus}$ and radius of $R_{\mathrm{p}}=1.69_{-0.08}^{+0.08} R_{\oplus}$ on a circular orbit with a period of $3.44 \mathrm{~d}$. We also derived a bulk density of $\rho_{\mathrm{p}}=6.7_{-1.1}^{+1.3} \mathrm{~g} \mathrm{~cm}^{-3}$ and an equilibrium temperature, assuming a zero albedo, of $T_{\text {eq }}=776 \pm 13 \mathrm{~K}$, which is slightly hotter than the mean surface temperature of Venus.

\subsection{Search for transit depth and time variations}

TESS observed TOI-1235 in three sectors and covered 22 transits of TOI-1235 b. This allowed us to assess the presence of transit-timing variations (TTVs) and transit depth variations. We carried out a search for TTVs using the batman package and fit each transit individually. We only left transit times and transit depth as free parameters, and fixed the remaining parameters to the values obtained in the joint analysis in Sect. 4.4. The best-fit parameters and associated uncertainties in our fitting procedure were derived using a Markov chain Monte Carlo analysis implemented in the emcee python package (Foreman-Mackey et al. 2013). We found an indication of a periodic TTV signal with a semiamplitude of about $4 \mathrm{~min}$. Using the GLS of the observed TTV signal, we found that the observed TTVs had a periodicity of $25.3 \pm 0.2 \mathrm{~d}$, which might indicate the presence of a second nontransiting planet in the system (Holman \& Murray 2005). However, a TTV signal with this amplitude might also easily be 
Table 5. Derived planetary parameters for TOI-1235 b.

\begin{tabular}{|c|c|}
\hline Parameter $^{(a)}$ & TOI-1235 b \\
\hline \multicolumn{2}{|c|}{ Derived transit parameters } \\
\hline$u_{1}^{(b)}$ & $0.38_{-0.24}^{+0.30}$ \\
\hline$u_{2}^{(b)}$ & $0.22_{-0.32}^{+0.35}$ \\
\hline$t_{T}(\mathrm{~h})$ & $2.094_{-0.086}^{+0.126}$ \\
\hline \multicolumn{2}{|c|}{ Derived physical parameters } \\
\hline$M_{\mathrm{p}}\left(M_{\oplus}\right)$ & $5.90_{-0.61}^{+0.62}$ \\
\hline$R_{\mathrm{p}}\left(R_{\oplus}\right)$ & $1.694_{-0.077}^{+0.080}$ \\
\hline$\rho_{\mathrm{p}}\left(\mathrm{g} \mathrm{cm}^{-3}\right)$ & $6.7_{-1.1}^{+1.3}$ \\
\hline$g_{\mathrm{p}}\left(\mathrm{m} \mathrm{s}^{-2}\right)$ & $20.1_{-2.7}^{+3.0}$ \\
\hline$a_{\mathrm{p}}(\mathrm{au})$ & $0.03826_{-0.00049}^{+0.00048}$ \\
\hline$T_{\text {eq }}(\mathrm{K})^{(c)}$ & $775_{-13}^{+13}$ \\
\hline$S\left(S_{\oplus}\right)$ & $60.3_{-1.5}^{+1.6}$ \\
\hline
\end{tabular}

Notes. ${ }^{(a)}$ Parameters obtained with the posterior values from Table 4 , $t_{\mathrm{T}}=$ Transit duration, from first contact to fourth contact. Error bars denote the $68 \%$ posterior credibility intervals. ${ }^{(b)}$ Derived from the TESS light curve. ${ }^{(c)}$ The equilibrium temperature was calculated assuming zero Bond albedo.

generated by the stellar activity (e.g., Oshagh et al. 2013), and the period was consistent with our previous analyses of the stellar rotation. We also searched for trends in the derived transit depths, and found that individual depths agreed within $1 \sigma$ with the depth derived from the combined analysis.

\section{Discussion}

Our 61 RV measurements yield a planetary mass for TOI-1235 b with an uncertainty of about $10 \%$, and the TESS and LCOGT light curves constrain the planetary radius at a level of about $5 \%$ uncertainty. This means that TOI- $1235 \mathrm{~b}$ belongs to the select group of terrestrial planets with a well-determined bulk density. The population with measurements better than $30 \%$ is shown in the mass-radius diagram of Fig. 9. The comparison of TOI-1235 b with theoretical models of Zeng et al. (2016, 2019) is consistent with a rocky $\mathrm{MgSiO}_{3}$-dominated composition with a bulk density slightly higher than that of Earth. This classifies it as a super-Earth planet.

Based again on the mass and radius relationships from Zeng et al. (2016), the best fit results in an iron core mass fraction of $\mathrm{CRF}=0.10_{-0.10}^{+0.38}$, but the planet is also consistent with an Earthlike bulk composition $(\mathrm{CRF} \approx 0.4-0.6)$. Furthermore, using Hardcore (Suissa et al. 2018) and our $R$ and $M$, the marginal core ratio fraction, $\mathrm{CRF}_{\text {marg }}$, is $0.53 \pm 0.20$, similar to the Earth's true CRF value of 0.55 .

Like many other transiting terrestrial and sub-Neptune planets, TOI- $1235 \mathrm{~b}$ is on a fairly irradiated orbit and therefore may have been strongly sculpted by extreme atmospheric escape due to XUV-driven photoevaporation (e.g., López \& Fortney 2013; Owen \& Wu 2013) or core-powered mass loss (e.g., Wu 2019; Gupta \& Schlichting 2020). Because of its expected low envelope mass fraction, the required binding energy makes this explanation difficult for TOI-1235 b, but using the escape scaling relations from López \& Fortney (2013), we found that this planet lies right at the boundary of where escape evolution is likely to play a significant role in removing primordial $\mathrm{H} / \mathrm{He}$ gaseous envelopes.
As described in Sect. 1 and illustrated by the insolationradius diagram in Fig. 9, the growing exoplanet statistics has revealed a gap in the radius distribution of planets slightly larger than Earth (Fulton et al. 2017). Rocky super-Earth planets of up to $\sim 1.5 R_{\oplus}$ are relatively common, as are gaseous mini-Neptunes in the range of $2-4 R_{\oplus}$, but only a few planets have been detected with a radius inside this gap (Gandolfi et al. 2019). Using the location of the radius valley as determined by Van Eylen et al. (2018), that is, $\log R=m \log P+a$ with $m=-0.09_{-0.04}^{+0.02}$ and $a=0.37_{-0.02}^{+0.04}$, we determine the predicted location of the radius valley at the orbital period of TOI- $1235 \mathrm{~b}$. We find that for $P=3.44 \mathrm{~d}$, the radius valley is located at $R=2.1 \pm 0.2 R_{\oplus}$. Therefore and according to this definition, TOI-1235 b, which has a radius $R=1.69_{-0.07}^{+0.08} R_{\oplus}$, would be located near the lower edge of the radius valley. Its rocky composition is indeed consistent with the planet having lost its atmosphere, as expected for planets below the radius valley (e.g., Owen \& Wu 2013).

However, the location of the radius gap as determined by Van Eylen et al. (2018) was based on F-, G-, and K-type stars, whereas TOI-1235 b orbits an M dwarf star. Whether these same boundaries apply to $\mathrm{M}$ dwarfs (and whether the gap actually exists for planets around $\mathrm{M}$ dwarfs) has been the subject of several recent studies (Zeng et al. 2017; Fulton \& Petigura 2018; Hirano et al. 2018). Following Zeng et al. (2017), for example, who used all of the Kepler planet candidates, the radius and stellar irradiation level of TOI-1235 b place it exactly in the gap for early-M dwarfs (located at about $1.7 R_{\oplus}$ for an irradiation of $60 S_{\oplus}$ in that work). On the other hand, when we extrapolate from the sample of Fulton \& Petigura (2018), who focused on F-, G-, and K-type stars with precise stellar parameters and on stars that host validated Kepler exoplanets, we reach a similar conclusion. Finally, using the sample of Hirano et al. (2018), who focused only on low-mass stars hosting validated small planets unveiled by K2 and Kepler, we would locate TOI-1235 b in the gap, but the data in that sample (arguably more suitable for a proper comparison with the stellar properties of TOI-1235) were unable to track a proper stellar irradiation versus radius dependence of the gap. Therefore our measurements of the bulk composition of TOI- $1235 \mathrm{~b}$, consistent with the planet having lost its atmosphere, place a strong constraint on any interpretation regarding the radius gap for $\mathrm{M}$ dwarfs at the irradiation levels received by TOI- $1235 \mathrm{~b}$. If atmospheric loss is indeed the correct physical interpretation for the radius gap, and if it applies to $\mathrm{M}$ dwarfs at the period or stellar irradiation level of TOI-1235 b, the gap for early-type $\mathrm{M}$ dwarfs has to be either at or above $1.7 R_{\oplus}$.

\section{Conclusions}

We confirmed that TOI-1235 $\mathrm{b}$ is a transiting super-Earth planet around an M0.5 V star, observed in sectors 14, 20, and 21 of the TESS mission. We collected CARMENES and HARPS-N spectroscopic data, from which we confirmed the planetary nature of the transit signal detected by TESS. Further support for the planetary interpretation came from our LCOGT photometric data during one transit, as well as from lucky and speckle imaging. From the joint analysis of all the data, we derived the following parameters for TOI-1235 b: mass of $M_{\mathrm{p}}=5.9 \pm 0.6 M_{\oplus}$, radius of $R_{\mathrm{p}}=1.69 \pm 0.08 R_{\oplus}$, and density of $\rho_{\mathrm{p}}=6.7_{-1.1}^{+1.7} \mathrm{~g} \mathrm{~cm}^{-3}$.

A comparison of the physical properties of TOI-1235 b with compositional models reveals the planet to be a rocky superEarth, with a bulk density only slightly higher than that of Earth. Although the location (and existence) of a radius gap 

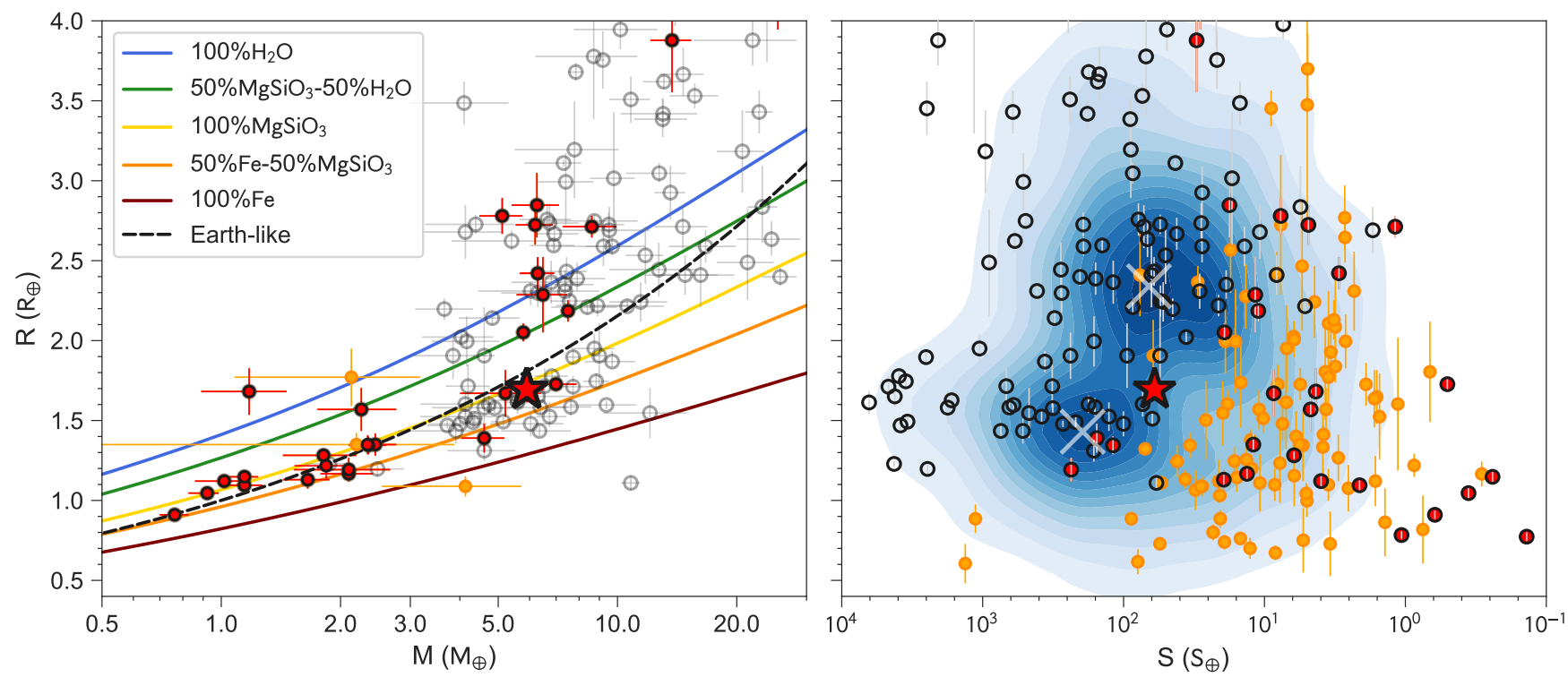

Fig. 9. Mass-radius (left) and insolation-radius (right) diagrams in Earth units. In the two panels, open circles are transiting planets around F-, G-, and K-type stars with mass and radius measurement better than 30\% from the TEPCat database of well-characterized planets (Southworth 2011), filled red circles are planets around $\mathrm{M}$ dwarfs with mass and radius measurement, filled yellow circles are planets around $\mathrm{M}$ dwarfs with mass determinations lower than $30 \%$ or without mass constraints at all (right panel only), and the red star is TOI-1235 b, whose radius and mass are determined with accuracies of 5 and $10 \%$, respectively. In the left panel, the color lines are the theoretical $R$ - $M$ models of Zeng et al. (2016), and the three planets with mass determinations lower than $30 \%$ are K2-3 b, BD-17 588A b, and LHS $1815 \mathrm{~b}$. In the right panel, we plot the $R-S$ point density of all the known confirmed transiting planets with contours, and mini-Neptunes and super-Earths density maxima with white crosses. The M dwarf without mass determination in the radius gap is K2-104 b (Mann et al. 2017), a planet around an active star in the Praesepe cluster that is fainter by 5 mag in $V$ than TOI-1235.

for exoplanets around M-dwarfs is still debated, the radius and irradiation level of TOI- $1235 \mathrm{~b}$ place it at the radius gap according to various suggestions of its location in the literature for these small low-mass stars. If the gap indeed exists for M-dwarfs, the bulk properties of TOI-1235 b, which make it consistent with having lost its atmosphere, constrain the gap to be at or above the planetary radius of TOI- $1235 \mathrm{~b}$, that is, $\sim 1.7 R_{\oplus}$ at its irradiation level $\left(\sim 60 S_{\oplus}\right)$. These findings help to better constrain the dependence of the gap location on stellar type and irradiation, and thus to understand its origin. Finally, the brightness of TOI-1235 ( $V \approx 11.5 \mathrm{mag}$ ) makes this planet an accessible and very interesting object for further studies of planet formation and atmospheric evolution.

Acknowledgements. CARMENES is an instrument for the Centro Astronómico Hispano-Alemán de Calar Alto (CAHA, Almería, Spain). CARMENES is funded by the German Max-Planck-Gesellschaft (MPG), the Spanish Consejo Superior de Investigaciones Científicas (CSIC), the European Union through FEDER/ERF FICTS-2011-02 funds, and the members of the CARMENES Consortium (Max-Planck-Institut für Astronomie, Instituto de Astrofísica de Andalucía, Landessternwarte Königstuhl, Institut de Ciències de l'Espai, Institut für Astrophysik Göttingen, Universidad Complutense de Madrid, Thüringer Landessternwarte Tautenburg, Instituto de Astrofísica de Canarias, Hamburger Sternwarte, Centro de Astrobiología and Centro Astronómico Hispano-Alemán) with additional contributions by the Spanish Ministry of Economy, the German Science Foundation through the Major Research Instrumentation Programme and DFG Research Unit FOR2544 "Blue Planets around Red Stars", the Klaus Tschira Stiftung, the states of Baden-Württemberg and Niedersachsen, and by the Junta de Andalucía. We acknowledge the use of public TESS Alert data from pipelines at the TESS Science Office and at the TESS Science Processing Operations Center. This research has made use of the Exoplanet Follow-up Observation Program website, which is operated by the California Institute of Technology, under contract with the National Aeronautics and Space Administration under the Exoplanet Exploration Program. Resources supporting this work were provided by the NASA High-End Computing (HEC) Program through the NASA Advanced Supercomputing (NAS) Division at Ames Research Center for the production of the SPOC data products. We acknowledge financial support from the European Research Council under the Horizon 2020 Framework Program via the
ERC Advanced Grant Origins 8324 28, the Deutsche Forschungsgemeinschaft through projects RE 281/32-1, RE 1664/14-1, RE 2694/4-1, and RA714/14-1, PA525/18-1, PA525/19-1 within the Schwerpunkt SPP 1992, the Agencia Estatal de Investigación of the Ministerio de Ciencia, Innovación y Universidades and the European FEDER/ERF funds through projects PGC2018-098153-B-C31, ESP2016-80435-C2-1-R, ESP2016-80435-C2-2-R, AYA2016-79425-C3-1/2/3P, AYA2015-69350-C3-2-P, RYC-2015-17697, and BES-2017-082610, the Centre of Excellence "Severo Ochoa" and "María de Maeztu" awards to the Instituto de Astrofísica de Canarias (SEV-2015-0548), Instituto de Astrofísica de Andalucía (SEV-2017-0709), and Centro de Astrobiología (MDM-2017-0737), the European Union's Horizon 2020 research and innovation program under the Marie Skłodowska-Curie grant 713673, the Centre national d'études spatiales through grants PLATO and GOLF, the Czech Academy of Sciences through grant LTT20015, NASA through grants NNX17AF27G and NNX17AG24G, JSPS KAKENHI through grants JP18H01265 and JP18H05439, JST PRESTO through grant JPMJPR 1775, the Fundación Bancaria "la Caixa" through grant INPhINIT LCF/BQ/IN17/11620033, and the Generalitat de Catalunya/CERCA programme. NESSI was funded by the NASA Exoplanet Exploration Program and the NASA Ames Research Center and built at the Ames Research Center. The authors are honored to be permitted to conduct observations on Iolkam Du'ag (Kitt Peak), a mountain within the Tohono O'odham Nation with particular significance to the Tohono O'odham people. This work made use of observations from the LCOGT network and the following software: astrasens, AstroImage], Banzai, batman, caracal, emcee, juliet, serval, TESS Transit Finder, tpfplotter, Yabi, and the python packages astropy, lightkurve, matplotlib, and numpy. We thank the SuperWASP team and J. Sanz-Forcada for sharing unpublished information with us. Special thanks to Ismael Pessa for all their support through this work.

\section{References}

Aller, A., Lillo-Box, J., Jones, D., Miranda, L. F., \& Barceló Forteza S. 2020, A\&A, 635, A128

Alonso-Floriano, F. J., Morales, J. C., Caballero, J. A., et al. 2015, A\&A, 577, A128

Arenou, F., Luri, X., Babusiaux, C., et al. 2018, A\&A, 616, A17

Astudillo-Defru, N., Delfosse, X., Bonfils, X., et al. 2017, A\&A, 600, A13

Astudillo-Defru, N., Cloutier, R., Wang, S. X., et al. 2020, A\&A, 636, A58

Bakos, G., Noyes, R. W., Kovács, G., et al. 2004, PASP, 116, 266

Barnes, R. 2017, Celes. Mech. Dyn. Astron., 129, 509 
Batalha, N. M., Rowe, J. F., Bryson, S. T., et al. 2013, ApJS, 204, 24

Bauer, F. F., Zechmeister, M., Kaminski, A., et al. 2020, ArXiv e-prints [arXiv:2006.01684]

Bayo, A., Rodrigo, C., Barrado Y Navascués, D., et al. 2008, A\&A, 492, 277

Berger, T. A., Huber, D., Gaidos, E., \& van Saders, J. L. 2018, ApJ, 866, 99

Bonfils, X., Delfosse, X., Udry, S., et al. 2013, A\&A, 549, A109

Boro Saikia, S., Marvin, C. J., Jeffers, S. V., et al. 2018, A\&A, 616, A108

Borsa, F., Scandariato, G., Rainer, M., et al. 2015, A\&A, 578, A64

Borucki, W. J. 2016, Rep. on Prog. Phys., 79, 036901

Borucki, W. J., Koch, D., Basri, G., et al. 2010, Science, 327, 977

Brown, T. M., Baliber, N., Bianco, F. B., et al. 2013, PASP, 125, 1031

Caballero, J. A., Guàrdia, J., López del Fresno, M., et al. 2016, SPIE Conf. Ser., 9910, 99100E

Cale, B., Plavchan, P., LeBrun, D., et al. 2019, AJ, 158, 170

Ceillier, T., Tayar, J., Mathur, S., et al. 2017, A\&A, 605, A111

Chabrier, G. 2003, PASP, 115, 763

Chen, H., \& Rogers, L. A. 2016, ApJ, 831, 180

Cifuentes, C., Caballero, J. A., Cortés-Contreras, M., et al. 2020, A\&A, submmited

Cloutier, R., Eastman, J. D., Rodriguez, J. E., et al. 2020a, AJ, 160, 3

Cloutier, R., Rodriguez, J. E., Irwin, J., et al. 2020b, AJ, 160, 22

Collins, K. A., Kielkopf, J. F., Stassun, K. G., \& Hessman, F. V. 2017, AJ, 153 77

Cosentino, R., Lovis, C., Pepe, F., et al. 2012, Proc. SPIE Conf. Ser., 8446, $84461 \mathrm{~V}$

Crossfield, I. J. M., Waalkes, W., Newton, E. R., et al. 2019, ApJ, 883, L16

Cutri, R. M., et al. 2014, VizieR Online Data Catalog: II/328

Díez Alonso, E., Caballero, J. A., Montes, D., et al. 2019, A\&A, 621, A126

Drake, A. J., Djorgovski, S. G., Mahabal, A., et al. 2009, ApJ, 696, 870

Dressing, C. D., \& Charbonneau, D. 2015, ApJ, 807, 45

Espinoza, N. 2018, Res. Notes Am. Astron. Soc., 2, 209

Espinoza, N., \& Jordán, A. 2016, MNRAS, 457, 3573

Espinoza, N., Kossakowski, D., \& Brahm, R. 2019, MNRAS, 490, 2262

Foreman-Mackey, D., Hogg, D. W., Lang, D., \& Goodman, J. 2013, PASP, 125, 306

Foreman-Mackey, D., Agol, E., Ambikasaran, S., \& Angus, R. 2017, celerite: Scalable 1D Gaussian Processes in $\mathrm{C}++$, Python, and Julia

Frith, J., Pinfield, D. J., Jones, H. R. A., et al. 2013, MNRAS, 435, 2161

Fulton, B. J., \& Petigura, E. A. 2018, AJ, 156, 264

Fulton, B. J., Petigura, E. A., Howard, A. W., et al. 2017, AJ, 154, 109

Gagné, J., Mamajek, E. E., Malo, L., et al. 2018, ApJ, 856, 23

Gaia Collaboration (Brown, A. G. A., et al.) 2018, A\&A, 616, A1

Gaidos, E., Mann, A. W., Lépine, S., et al. 2014, MNRAS, 443, 2561

Gaidos, E., Mann, A. W., Kraus, A. L., \& Ireland, M. 2016, MNRAS, 457, 2877

Gan, T., Shporer, A., Livingston, J. H., et al. 2020, AJ, 159, 160

Gandolfi, D., Fossati, L., Livingston, J. H., et al. 2019, ApJ, 876, L24

García, R. A., \& Ballot, J. 2019, Liv. Rev. Sol. Phys., 16, 4

García, R. A., Hekker, S., Stello, D., et al. 2011, MNRAS, 414, L6

García, R. A., Mathur, S., Pires, S., et al. 2014a, A\&A, 568, A10

García, R. A., Ceillier, T., Salabert, D., et al. 2014b, A\&A, 572, A34

Gilbert, E. A., Barclay, T., Schlieder, J. E., et al. 2020, AJ, submitted [arXiv:2001.00952]

Ginzburg, S., Schlichting, H. E., \& Sari, R. 2016, ApJ, 825, 29

Ginzburg, S., Schlichting, H. E., \& Sari, R. 2018, MNRAS, 476, 759

Günther, M. N., Pozuelos, F. J., Dittmann, J. A., et al. 2019, Nat. Astron., 3, 1099

Gupta, A., \& Schlichting, H. E. 2019, MNRAS, 487, 24

Gupta, A., \& Schlichting, H. E. 2020, MNRAS, 493, 792

Heinze, A. N., Tonry, J. L., Denneau, L., et al. 2018, AJ, 156, 241

Henry, T. J., Jao, W.-C., Subasavage, J. P., et al. 2006, AJ, 132, 2360

Hirano, T., Dai, F., Gandolfi, D., et al. 2018, AJ, 155, 127

Høg, E., Fabricius, C., Makarov, V. V., et al. 2000, A\&A, 355, L27

Holman, M. J., \& Murray, N. W. 2005, Science, 307, 1288

Hormuth, F., Brandner, W., Hippler, S., \& Henning, T. 2008, J. Phys. Conf. Ser. 131, 012051

Howell, S. B., Everett, M. E., Sherry, W., Horch, E., \& Ciardi, D. R. 2011, AJ, 142,19

Hunter, A., Macgregor, A., Szabo, T., Wellington, C., \& Bellgard, M. 2012, Source Code Biol. Med., 7, 1

Irwin, J., Berta, Z. K., Burke, C. J., et al. 2011, ApJ, 727, 56

Jeffers, S. V., Schöfer, P., Lamert, A., et al. 2018, A\&A, 614, A76

Jenkins, J. M. 2002, ApJ, 575, 493

Jenkins, J. M., Twicken, J. D., McCauliff, S., et al. 2016, Proc. SPIE, 9913, 99133E

Jenkins, J. M., Tenenbaum, P., Seader, S., et al. 2017, Kepler Data Processing Handbook: Transiting Planet Search (USA: Kepler Science Document)

Jensen, E. 2013, Astrophysics Source Code Library [record ascl : 1306.007]

Jin, S., Mordasini, C., Parmentier, V., et al. 2014, ApJ, 795, 65

Johnson, D. R. H., \& Soderblom, D. R. 1987, AJ, 93, 864
Kaminski, A., Trifonov, T., Caballero, J. A., et al. 2018, A\&A, 618, A115 Kipping, D. M. 2013, MNRAS, 435, 2152

Kirkpatrick, J. D., Kellogg, K., Schneider, A. C., et al. 2016, ApJS, 224, 36 Kochanek, C. S., Shappee, B. J., Stanek, K. Z., et al. 2017, PASP, 129, 104502 Kreidberg, L. 2015, PASP, 127, 1161

Lafarga, M., Ribas, I., Lovis, C., et al. 2020, A\&A, 636, A36

Lépine, S., \& Gaidos, E. 2011, AJ, 142, 138

Lépine, S., \& Shara, M. M. 2005, AJ, 129, 1483

Lépine, S., Hilton, E. J., Mann, A. W., et al. 2013, AJ, 145, 102

Li, J., Tenenbaum, P., Twicken, J. D., et al. 2019, PASP, 131, 024506

Lillo-Box, J., Barrado, D., \& Bouy, H. 2012, A\&A, 546, A10

Lindegren, L., Hernández, J., Bombrun, A., et al. 2018, A\&A, 616, A2

Livingston, J. H., Endl, M., Dai, F., et al. 2018, AJ, 156, 78

López, E. D., \& Fortney, J. J. 2013, ApJ, 776, 2

López, E. D., \& Fortney, J. J. 2014, ApJ, 792, 1

Luque, R., Pallé, E., Kossakowski, D., et al. 2019, A\&A, 628, A39

Mann, A. W., Gaidos, E., Vanderburg, A., et al. 2017, AJ, 153, 64

Martínez-Rodríguez, H., Caballero, J. A., Cifuentes, C., Piro, A. L., \& Barnes, R. 2019, ApJ, 887, 261

Mathur, S., García, R. A., Régulo, C., et al. 2010, A\&A, 511, A46

McCully, C., Volgenau, N. H., Harbeck, D.-R., et al. 2018, Proc. SPIE Conf. Ser., 10707, 107070K

McQuillan, A., Mazeh, T., \& Aigrain, S. 2014, ApJS, 211, 24

Montes, D., González-Peinado, R., Tabernero, H. M., et al. 2018, MNRAS, 479, 1332

Morbidelli, A. 2018, ArXiv e-prints [arXiv:1803.06708]

Mortier, A., \& Collier Cameron, A. 2017, A\&A, 601, A110

Mortier, A., Faria, J. P., Correia, C. M., Santerne, A., \& Santos, N. C. 2015, A\&A, 573, A101

Mulders, G. D., Pascucci, I., \& Apai, D. 2015, ApJ, 798, 112

Nava, C., López-Morales, M., Haywood, R. D., \& Giles, H. A. C. 2020, AJ, 159, 23

Nowak, G., Luque, R., Parviainen, H., et al. 2020, A\&A, submitted [arXiv:2003.01140]

Oshagh, M., Santos, N. C., Boisse, I., et al. 2013, A\&A, 556, A19

Owen, J. E., \& Wu, Y. 2013, ApJ, 775, 105

Owen, J. E., \& Wu, Y. 2017, ApJ, 847, 29

Passegger, V. M., Schweitzer, A., Shulyak, D., et al. 2019, A\&A, 627, A161

Perger, M., Scandariato, G., Ribas, I., et al. 2019, A\&A, 624, A123

Pojmański, G. 1997, Acta Astron., 47, 467

Pollacco, D. L., Skillen, I., Collier Cameron, A., et al. 2006, PASP, 118, 1407

Quirrenbach, A., Amado, P. J., Caballero, J. A., et al. 2014, Proc. SPIE, 9147, 91471F

Quirrenbach, A., Amado, P. J., Ribas, I., et al. 2018, SPIE, 10702, 107020W

Rayner, J., Tokunaga, A., Jaffe, D., et al. 2016, Proc. SPIE Conf. Ser., 9908, 990884

Reiners, A., Zechmeister, M., Caballero, J. A., et al. 2018, A\&A, 612, A49

Ricker, G. R., Winn, J. N., Vanderspek, R., et al. 2015, J. Astron. Teles. Instrum. Syst., 1, 014003

Santos, A. R. G., García, R. A., Mathur, S., et al. 2019, ApJS, 244, 21

Schöfer, P., Jeffers, S. V., Reiners, A., et al. 2019, A\&A, 623, A44

Schweitzer, A., Passegger, V. M., Cifuentes, C., et al. 2019, A\&A, 625, A68

Scott, N. J., \& Howell, S. B. 2018, Proc. SPIEConf. Ser., 10701, 107010G

Scott, N. J., Howell, S. B., Horch, E. P., \& Everett, M. E. 2018, PASP, 130, 054502

Shappee, B. J., Prieto, J. L., Grupe, D., et al. 2014, ApJ, 788, 48

Skrutskie, M. F., Cutri, R. M., Stiening, R., et al. 2006, AJ, 131, 1163

Smith, J. C., Stumpe, M. C., Van Cleve, J. E., et al. 2012, PASP, 124, 1000

Southworth, J. 2011, MNRAS, 417, 2166

Stassun, K. G., Oelkers, R. J., Pepper, J., et al. 2018, AJ, 156, 102

Stassun, K. G., Oelkers, R. J., Paegert, M., et al. 2019, AJ, 158, 138

Strehl, K. 1902, Astron. Nachri., 158, 89

Stumpe, M. C., Smith, J. C., Van Cleve, J. E., et al. 2012, PASP, 124, 985

Stumpe, M. C., Smith, J. C., Catanzarite, J. H., et al. 2014, PASP, 126, 100

Suissa, G., Chen, J., \& Kipping, D. 2018, MNRAS, 476, 2613

Tal-Or, L., Trifonov, T., Zucker, S., Mazeh, T., \& Zechmeister, M. 2019, MNRAS, 484, L8

Torrence, C., \& Compo, G. P. 1998, Bull. Am. Meteorol. Soc., 79, 61

Trifonov, T., Kürster, M., Zechmeister, M., et al. 2018, A\&A, 609, A117

Trifonov, T., Tal-Or, L., Zechmeister, M., et al. 2020, A\&A, 636, A74

Twicken, J. D., Catanzarite, J. H., Clarke, B. D., et al. 2018, PASP, 130, 064502

Van Eylen, V., Agentoft, C., Lundkvist, M. S., et al. 2018, MNRAS, 479, 4786

Winn, J. N., Petigura, E. A., Morton, T. D., et al. 2017, AJ, 154, 270

Woźniak, P. R., Vestrand, W. T., Akerlof, C. W., et al. 2004, AJ, 127, 2436

Wright, J. T., Marcy, G. W., Butler, R. P., \& Vogt, S. S. 2004, ApJS, 152, 261

Wu, Y. 2019, ApJ, 874, 91

Zechmeister, M., \& Kürster, M. 2009, A\&A, 496, 577

Zechmeister, M., Anglada-Escudé, G., \& Reiners, A. 2014, A\&A, 561, A59 
Zechmeister, M., Reiners, A., Amado, P. J., et al. 2018, A\&A, 609, A12 Zeng, L., Sasselov, D. D., \& Jacobsen, S. B. 2016, ApJ, 819, 127

Zeng, L., Jacobsen, S. B., \& Sasselov, D. D. 2017, Res. Notes Am. Astron. Soc., 1,32

Zeng, L., Jacobsen, S. B., Sasselov, D. D., et al. 2019, Proc. Natl. Acad. Sci., 116, 9723

${ }^{1}$ Landessternwarte, Zentrum für Astronomie der Universität Heidelberg, Königstuhl 12, 69117 Heidelberg, Germany e-mail: pbluhm@lsw.uni-heidelberg.de

2 Instituto de Astrofísica de Canarias, 38205 La Laguna, Tenerife, Spain

${ }^{3}$ Departamento de Astrofísica, Universidad de La Laguna, 38206 La Laguna, Tenerife, Spain

${ }^{4}$ Space Telescope Science Institute, 3700 San Martin Drive, Baltimore, MD 21218, USA

5 Centro de Astrobiología (CSIC-INTA), ESAC, Camino bajo del castillo s/n, 28692 Villanueva de la Cañada, Madrid, Spain

${ }^{6}$ Instituto de Astrofísica de Andalucía (CSIC), Glorieta de la Astronomía s/n, 18008 Granada, Spain

${ }^{7}$ Institut für Astrophysik, Georg-August-Universität, FriedrichHund-Platz 1, 37077 Göttingen, Germany

${ }^{8}$ Department of Astronomy, University of Tokyo, 7-3-1 Hongo, Bunkyo-ky, Tokyo 113-0033, Japan

${ }^{9}$ Mullard Space Science Laboratory, University College London, Holmbury St. Mary, Dorking, Surrey, RH5 6NT, UK

${ }^{10}$ NASA Goddard Space Flight Center, 8800 Greenbelt Road, Greenbelt, MD 20771, USA

11 Sellers Exoplanet Environments Collaboration, NASA Goddard Space Flight Center, Greenbelt, MD 20771, USA

12 Deutsches Zentrum für Luft- und Raumfahrt, Institut für Planetenforschung, 12489 Berlin, Rutherfordstrasse 2., Germany

13 Centro de Astrobiología (CSIC-INTA), Carretera de Ajalvir km 4, 28850 Torrejón de Ardoz, Madrid, Spain

14 Department of Physics and Astronomy, George Mason University, 4400 University Drive, Fairfax, VA 22030, USA

15 Thüringer Landessternwarte Tautenburg, Sternwarte 5, 07778 Tautenburg, Germany

${ }^{16}$ Center for Planetary Systems Habitability and McDonald Observatory, The University of Texas at Austin, Austin, TX 78730, USA

17 Center for Astrophysics I Harvard \& Smithsonian, 60 Garden Street, Cambridge, MA 02138, USA

18 Departamento de Explotación y Prospección de Minas, Escuela de Minas, Energía y Materiales, Universidad de Oviedo, 33003 Oviedo, Spain

19 Instituto Universitario de Ciencias y Tecnologías del Espacio de Asturias, Independencia 13, 33004 Oviedo, Spain

${ }^{20}$ Department of Physics, University of Khartoum, Al-Gamaa Ave, Khartoum 11111, Sudan

${ }^{21}$ Campo Catino Astronomical Observatory, Regione Lazio, 03010 Guarcino (FR), Italy

${ }^{22}$ Leiden Observatory, Leiden University, 2333CA Leiden, The Netherlands

23 Department of Space, Earth and Environment, Chalmers University of Technology, Onsala Space Observatory, 43992 Onsala, Sweden

${ }^{24}$ Department of Earth Sciences, University of Hawai'i at Māanoa, Honolulu, HI 96822 USA
${ }^{25}$ IRFU, CEA, Université Paris-Saclay, 91191 Gif-sur-Yvette, France

26 AIM, CEA, CNRS, Université Paris-Saclay, Université Paris Diderot, Sorbonne Paris Cité, 91191 Gif-sur-Yvette, France

27 Observatori Astronòmic Albanyà, Camí de Bassegoda s/n, 17733 Albanyà, Girona, Spain

28 Max-Planck-Institut für Astronomie, Königstuhl 17, 69117 Heidelberg, Germany

${ }^{29}$ Institut de Ciències de l'Espai (ICE, CSIC), Campus UAB, Can Magrans s/n, 08193 Bellaterra, Spain

${ }^{30}$ Institut d'Estudis Espacials de Catalunya (IEEC), 08034 Barcelona, Spain

31 NASA 31 Research Center, Moffett Field, CA 94035, USA

32 Department of Physics \& Astronomy, Swarthmore College, Swarthmore PA 19081, USA

33 Astronomical Institute, Czech Academy of Sciences, Fričova 298, 25165, Ondřejov, Czech Republic

34 Rheinisches Institut für Umweltforschung an der Universität $\mathrm{zu}$ Köln, Aachener Strasse 209, 50931 Köln, Germany

35 Departamento de Física de la Tierra y Astrofísica and IPARCOSUCM (Instituto de Física de Partículas y del Cosmos de la UCM), Facultad de Ciencias Físicas, Universidad Complutense de Madrid, 28040, Madrid, Spain

${ }^{36}$ Komaba Institute for Science, The University of Tokyo, 3-8-1 Komaba, Meguro, Tokyo 153-8902, Japan

37 JST, PRESTO, 3-8-1 Komaba, Meguro, Tokyo 153-8902, Japan

38 Astrobiology Center, 2-21-1 Osawa, Mitaka, Tokyo 181-8588, Japan

39 Hamburger Sternwarte, Universität Hamburg, Gojenbergsweg 112, 21029 Hamburg, Germany

40 Homer L. Dodge Department of Physics and Astronomy, University of Oklahoma, 440 West Brooks Street, Norman, OK 73019, USA

41 Centro Astronómico Hispano-Alemán, Observatorio de Calar Alto, Sierra de los Filabres, 04550 Gérgal, Spain

42 Zentrum für Astronomie und Astrophysik, Technische Universität Berlin, Hardenbergstr. 36, 10623 Berlin, Germany

43 Institut für Geologische Wissenschaften, Freie Universität Berlin, Malteserstr. 74-100, 12249 Berlin, Germany

44 Astronomy Department and Van Vleck Observatory, Wesleyan University, Middletown, CT 06459, USA

45 Kavli Institute for Astrophysics and Space Research, Massachusetts Institute of Technology, Cambridge, MA 02139, USA

46 Space Science Institute, 4765 Walnut St., Suite B, Boulder, CO 80301, USA

47 Department of Earth, Atmospheric and Planetary Sciences, Massachusetts Institute of Technology, Cambridge, MA 02139, USA

48 Department of Aeronautics and Astronautics, Massachusetts Institute of Technology, 77 Massachusetts Avenue, Cambridge, MA 02139, USA

49 School of Physics and Astronomy, Queen Mary University London, 327 Mile End Road, London E1 4NS, UK

50 Department of Physics, Ariel University, Ariel 40700, Israel

51 Vereniging Voor Sterrenkunde, Brugge, Belgium \& Centre for mathematical Plasma-Astrophysics, Department of Mathematics, KU Leuven, Celestijnenlaan 200B, 3001 Heverlee, Belgium

52 AstroLAB IRIS, Provinciaal Domein "De Palingbeek", Verbrandemolenstraat 5, 8902 Zillebeke, Ieper, Belgium

53 Department of Physics and Astronomy, Mississippi State University, 355 Lee Boulevard, Mississippi State, MS 39762, USA 


\section{Appendix A: Long tables}

Table A.1. Priors used for TOI-1235 b in the joint fit with juliet.

\begin{tabular}{|c|c|c|c|}
\hline Parameter $^{(a)}$ & Prior & Unit & Description \\
\hline \multicolumn{4}{|c|}{ Stellar parameters } \\
\hline$\rho_{\star}$ & $\mathcal{N}(3.7,3.8)$ & $\mathrm{g} \mathrm{cm}^{-3}$ & Stellar density \\
\hline \multicolumn{4}{|c|}{ Planet parameters } \\
\hline$P_{\mathrm{b}}$ & $\mathcal{U}(3,4)$ & d & Period of planet $b$ \\
\hline$t_{0, b}$ & $\mathcal{U}(2458683,2458687)$ & $\mathrm{d}$ & Time of transit center of planet $b$ \\
\hline$r_{1, b}$ & $\mathcal{U}(0,1)$ & $\ldots$ & Parameterization for $p$ and $b$ \\
\hline$r_{2, b}$ & $\mathcal{U}(0,1)$ & $\ldots$ & Parameterization for $p$ and $b$ \\
\hline$K_{b}$ & $\mathcal{N}(0,100)$ & $\mathrm{ms}^{-1}$ & RV semi-amplitude of planet $b$ \\
\hline$e_{b}$ & 0.0 (fixed) & $\ldots$ & Orbital eccentricity of planet $b$ \\
\hline$\omega_{b}$ & 90.0 (fixed) & $\operatorname{deg}$ & Periastron angle of planet $b$ \\
\hline \multicolumn{4}{|c|}{ Photometry parameters } \\
\hline$D_{\text {TESS }}$ & 1.0 (fixed) & $\ldots$ & Dilution factor for TESS Sectors 14, 20, and 21 \\
\hline$M_{\mathrm{TESS}, \mathrm{S} 14}$ & $\mathcal{N}(0,0.1)$ & $\ldots$ & Relative flux offset for TESS Sector 14 \\
\hline$M_{\mathrm{TESS}, \mathrm{S} 20}$ & $\mathcal{N}(0,0.1)$ & $\ldots$ & Relative flux offset for TESS Sector 20 \\
\hline$M_{\mathrm{TESS}, \mathrm{S} 21}$ & $\mathcal{N}(0,0.1)$ & $\ldots$ & Relative flux offset for TESS Sector 21 \\
\hline$\sigma_{\mathrm{TESS}, \mathrm{S} 14}$ & $\mathcal{L} \mathcal{U}\left(1,10^{4}\right)$ & ppm & Extra jitter term for TESS Sector 14 \\
\hline$\sigma_{\mathrm{TESS}, \mathrm{S} 20}$ & $\mathcal{L} \mathcal{U}\left(1,10^{4}\right)$ & ppm & Extra jitter term for TESS Sector 20 \\
\hline$\sigma_{\mathrm{TESS}, \mathrm{S} 21}$ & $\mathcal{L} \mathcal{U}\left(1,10^{4}\right)$ & ppm & Extra jitter term TESS Sector 21 \\
\hline$q_{1, \mathrm{TESS}}$ & $\mathcal{U}(0,1)$ & $\ldots$ & Limb-darkening parameterization for TESS Sectors $14,20,21$ \\
\hline$q_{2, \mathrm{TESS}}$ & $\mathcal{U}(0,1)$ & $\ldots$ & Limb-darkening parameterization for TESS Sectors $14,20,21$ \\
\hline$D_{\text {LCO }}$ & 1.0 (fixed) & $\ldots$ & Dilution factor for LCOGT \\
\hline$q_{1, \mathrm{LCO}}$ & $\mathcal{U}(0,1)$ & $\ldots$ & Limb-darkening parameterization for LCOGT \\
\hline$M_{\mathrm{LCO}}$ & $\mathcal{N}(0,0.1)$ & $\ldots$ & Relative flux offset for LCOGT \\
\hline$\sigma_{\mathrm{LCO}}$ & $\mathcal{L U}(1,10000)$ & ppm & Extra jitter term for LCOGT \\
\hline$\theta_{0, \mathrm{LCO}}$ & $\mathcal{U}(-100,100)$ & $\ldots$ & Extra jitter term for LCOGT \\
\hline$\theta_{1, \mathrm{LCO}}$ & $\mathcal{U}(-100,100)$ & $\ldots$ & Extra jitter term for LCOGT \\
\hline \multicolumn{4}{|c|}{$R V$ parameters } \\
\hline$\gamma_{\text {HARPS-N }}$ & $\mathcal{N}(0,10)$ & $\mathrm{m} \mathrm{s}^{-1}$ & RV zero-point for HARPS-N \\
\hline$\sigma_{\text {HARPS-N }}$ & $\mathcal{L U}(0.01,10)$ & $\mathrm{m} \mathrm{s}^{-1}$ & Extra jitter term for HARPS-N \\
\hline$\gamma_{\text {CARMENES }}$ & $\mathcal{N}(0,10)$ & $\mathrm{m} \mathrm{s}^{-1}$ & RV zero point for CARMENES \\
\hline$\sigma_{\text {CARMENES }}$ & $\mathcal{L} \mathcal{U}(0.01,10)$ & $\mathrm{m} \mathrm{s}^{-1}$ & Extra jitter term for CARMENES \\
\hline \multicolumn{4}{|c|}{ GP hyperparameters } \\
\hline$\sigma_{\mathrm{GP}, \mathrm{RV}}$ & $\mathcal{L} \mathcal{U}\left(10^{-10}, 100\right)$ & $\mathrm{m} \mathrm{s}^{-1}$ & Amplitude of GP component for the RVs \\
\hline$\alpha_{\mathrm{GP}, \mathrm{RV}}$ & $\mathcal{L} \mathcal{U}\left(10^{-10}, 100\right)$ & $\mathrm{d}^{-2}$ & Inverse length-scale of GP exponential component for the RVs \\
\hline$\Gamma_{\mathrm{GP}, \mathrm{RV}}$ & $\mathcal{L} \mathcal{U}\left(10^{-10}, 100\right)$ & $\ldots$ & Amplitude of GP sine-squared component for the RVs \\
\hline$P_{\text {rot;GP,RV }}$ & $\mathcal{U}(1,100)$ & $\mathrm{d}$ & Period of the GP quasi-periodic component for the RVs \\
\hline
\end{tabular}

Notes. ${ }^{(a)}$ The parameterization for $(p, b)$ was made with $\left(r_{1}, r_{2}\right)$ as in Espinoza (2018). The prior labels of $\mathcal{N}, \mathcal{U}$, and $\mathcal{L} \mathcal{U}$ represent normal, uniform, and log-uniform distributions, respectively, where $\mathcal{N}\left(\mu, \sigma^{2}\right)$ is a normal distribution of the mean $\mu$ and variance $\sigma^{2}$ and $\mathcal{U}(a, b)$ and $\mathcal{L} \mathcal{U}(a, b)$ are uniform and log-uniform distributions between $a$ and $b$. 
A\&A 639, A132 (2020)

Table A.2. Radial velocity measurements and spectroscopic activity indicators for TOI-1235 from optical spectra.

\begin{tabular}{|c|c|c|c|c|c|c|c|c|}
\hline \multicolumn{9}{|c|}{ CARMENES VIS } \\
\hline $\begin{array}{l}\text { BJD } \\
(-2450000)\end{array}$ & $\begin{array}{c}\mathrm{RV} \\
\left(\mathrm{m} \mathrm{s}^{-1}\right)\end{array}$ & $\begin{array}{c}\mathrm{CRX} \\
\left(\mathrm{m} \mathrm{s}^{-1} \mathrm{~Np}^{-1}\right)\end{array}$ & $\begin{array}{c}\mathrm{dLW} \\
\left(\mathrm{m}^{2} \mathrm{~s}^{-2}\right)\end{array}$ & $\mathrm{H} \alpha$ & Ca IRTa & $\mathrm{TiO} 7050$ & $\mathrm{TiO} 8430$ & TiO8860 \\
\hline 8796.6533 & $-1.2 \pm 2.2$ & $9 \pm 19$ & $-30.4 \pm 3.8$ & $0.6954 \pm 0.0021$ & $0.5204 \pm 0.0022$ & $0.8474 \pm 0.0015$ & $0.8656 \pm 0.0027$ & $0.9722 \pm 0.0028$ \\
\hline 8807.7240 & $0.4 \pm 2.4$ & $-24 \pm 20$ & $-3.2 \pm 2.8$ & $0.7004 \pm 0.0017$ & $0.5221 \pm 0.0018$ & $0.8487 \pm 0.0012$ & $0.8694 \pm 0.0022$ & $0.9684 \pm 0.0023$ \\
\hline 8811.6588 & $6.3 \pm 2.8$ & $37 \pm 25$ & $-22.0 \pm 4.1$ & $0.6859 \pm 0.0027$ & $0.5194 \pm 0.0029$ & $0.8515 \pm 0.0020$ & $0.8640 \pm 0.0036$ & $0.9746 \pm 0.0035$ \\
\hline 8815.7134 & $-2.7 \pm 1.8$ & $8 \pm 15$ & $-0.8 \pm 2.0$ & $0.6907 \pm 0.0014$ & $0.5294 \pm 0.0016$ & $0.8494 \pm 0.0011$ & $0.8665 \pm 0.0019$ & $0.9742 \pm 0.0020$ \\
\hline 8816.6576 & $3.0 \pm 1.6$ & $-14 \pm 13$ & $5.5 \pm 2.0$ & $0.6916 \pm 0.0013$ & $0.5299 \pm 0.0015$ & $0.8859 \pm 0.0010$ & $0.8650 \pm 0.0018$ & $0.9768 \pm 0.0018$ \\
\hline 8817.7185 & $0.4 \pm 3.2$ & $64 \pm 29$ & $-19.3 \pm 3.4$ & $0.6973 \pm 0.0030$ & $0.5223 \pm 0.0035$ & $0.8379 \pm 0.0022$ & $0.8542 \pm 0.0042$ & $0.9759 \pm 0.0042$ \\
\hline 8831.5414 & $5.4 \pm 2.8$ & $0 \pm 23$ & $-4.6 \pm 3.3$ & $0.6884 \pm 0.0020$ & $0.5199 \pm 0.0022$ & $0.8460 \pm 0.0015$ & $0.8667 \pm 0.0027$ & $0.9770 \pm 0.0027$ \\
\hline 8832.6949 & $2.5 \pm 2.2$ & $-7 \pm 19$ & $11.4 \pm 3.3$ & $0.6893 \pm 0.0018$ & $0.5223 \pm 0.0020$ & $0.8486 \pm 0.0014$ & $0.8629 \pm 0.0025$ & $0.9782 \pm 0.0025$ \\
\hline 8846.6694 & $-4.2 \pm 1.6$ & $16 \pm 14$ & $6.3 \pm 1.6$ & $0.6894 \pm 0.0011$ & $0.5267 \pm 0.0013$ & $0.8514 \pm 0.0009$ & $0.8666 \pm 0.0016$ & $0.9769 \pm 0.0017$ \\
\hline 8848.7121 & $5.8 \pm 1.6$ & $27 \pm 13$ & $10.1 \pm 1.6$ & $0.6963 \pm 0.0011$ & $0.5200 \pm 0.0013$ & $0.8489 \pm 0.0008$ & $0.8640 \pm 0.0015$ & $0.9765 \pm 0.0016$ \\
\hline 8850.6431 & $2.8 \pm 1.4$ & $12 \pm 13$ & $1.5 \pm 1.9$ & $0.6905 \pm 0.0012$ & $0.5256 \pm 0.0014$ & $0.8518 \pm 0.0009$ & $0.8709 \pm 0.0017$ & $0.9761 \pm 0.0017$ \\
\hline 8852.6259 & $4.5 \pm 1.4$ & $9 \pm 12$ & $8.0 \pm 1.3$ & $0.6989 \pm 0.0012$ & $0.5191 \pm 0.0014$ & $0.8502 \pm 0.0009$ & $0.8687 \pm 0.0017$ & $0.9812 \pm 0.0017$ \\
\hline 8854.6620 & $6.0 \pm 2.1$ & $27 \pm 14$ & $5.5 \pm 1.8$ & $0.6950 \pm 0.0011$ & $0.5268 \pm 0.0013$ & $0.8488 \pm 0.0009$ & $0.8647 \pm 0.0016$ & $0.9798 \pm 0.0016$ \\
\hline 8855.6361 & $4.7 \pm 2.0$ & $-3 \pm 14$ & $2.5 \pm 1.6$ & $0.6864 \pm 0.0012$ & $0.5254 \pm 0.0013$ & $0.8499 \pm 0.0009$ & $0.8678 \pm 0.0016$ & $0.9754 \pm 0.0016$ \\
\hline 8856.6278 & $0.0 \pm 1.5$ & $13 \pm 11$ & $6.6 \pm 1.7$ & $0.6868 \pm 0.0011$ & $0.5187 \pm 0.0013$ & $0.8523 \pm 0.0009$ & $0.8650 \pm 0.0015$ & $0.9756 \pm 0.0016$ \\
\hline 8857.6312 & $-1.5 \pm 1.9$ & $6 \pm 13$ & $6.1 \pm 2.1$ & $0.6952 \pm 0.0011$ & $0.5283 \pm 0.0013$ & $0.8491 \pm 0.0009$ & $0.8666 \pm 0.0016$ & $0.9832 \pm 0.0016$ \\
\hline 8858.6017 & $6.7 \pm 2.0$ & $15 \pm 16$ & $-2.3 \pm 2.5$ & $0.6934 \pm 0.0017$ & $0.5265 \pm 0.0018$ & $0.8514 \pm 0.0013$ & $0.8659 \pm 0.0022$ & $0.9826 \pm 0.0023$ \\
\hline 8860.6327 & $-3.8 \pm 1.8$ & $-3 \pm 16$ & $2.8 \pm 2.3$ & $0.6859 \pm 0.0015$ & $0.5227 \pm 0.0017$ & $0.8538 \pm 0.0012$ & $0.8665 \pm 0.0020$ & $0.9769 \pm 0.0021$ \\
\hline 8861.6279 & $-3.8 \pm 1.9$ & $26 \pm 15$ & $3.2 \pm 2.3$ & $0.6944 \pm 0.0014$ & $0.5262 \pm 0.0015$ & $0.8506 \pm 0.0010$ & $0.8665 \pm 0.0019$ & $0.9794 \pm 0.0019$ \\
\hline 8862.6304 & $-0.2 \pm 1.8$ & $27 \pm 14$ & $-0.7 \pm 1.9$ & $0.6947 \pm 0.0014$ & $0.5285 \pm 0.0016$ & $0.8509 \pm 0.0011$ & $0.8693 \pm 0.0019$ & $0.9768 \pm 0.0020$ \\
\hline 8863.6852 & $-1.4 \pm 3.0$ & $-48 \pm 27$ & $2.7 \pm 3.5$ & $0.6932 \pm 0.0021$ & $0.5312 \pm 0.0023$ & $0.8470 \pm 0.0016$ & $0.8613 \pm 0.0028$ & $0.9778 \pm 0.0029$ \\
\hline 8864.6148 & $-3.8 \pm 2.1$ & $-11 \pm 16$ & $-6.6 \pm 2.4$ & $0.6862 \pm 0.0016$ & $0.5217 \pm 0.0018$ & $0.8507 \pm 0.0012$ & $0.8662 \pm 0.0021$ & $0.9758 \pm 0.0021$ \\
\hline 8865.6228 & $0.6 \pm 2.5$ & $-33 \pm 23$ & $-5.5 \pm 2.6$ & $0.6967 \pm 0.0019$ & $0.5277 \pm 0.0021$ & $0.8477 \pm 0.0014$ & $0.8648 \pm 0.0025$ & $0.9864 \pm 0.0026$ \\
\hline 8866.6362 & $2.0 \pm 3.4$ & $-23 \pm 28$ & $-4.4 \pm 3.5$ & $0.6948 \pm 0.0028$ & $0.5287 \pm 0.0030$ & $0.8479 \pm 0.0020$ & $0.8626 \pm 0.0036$ & $0.9867 \pm 0.0036$ \\
\hline 8877.5779 & $-2.4 \pm 2.0$ & $-10 \pm 13$ & $-4.4 \pm 2.2$ & $0.6927 \pm 0.0011$ & $0.5219 \pm 0.0012$ & $0.8467 \pm 0.0008$ & $0.8682 \pm 0.0015$ & $0.9839 \pm 0.0015$ \\
\hline 8881.5843 & $-0.6 \pm 1.6$ & $13 \pm 15$ & $-8.0 \pm 1.9$ & $0.6875 \pm 0.0012$ & $0.5185 \pm 0.0013$ & $0.8471 \pm 0.0009$ & $0.8670 \pm 0.0016$ & $0.9821 \pm 0.0017$ \\
\hline 8882.5742 & $0.3 \pm 1.6$ & $2 \pm 14$ & $-19.2 \pm 2.2$ & $0.6948 \pm 0.0014$ & $0.5208 \pm 0.0015$ & $0.8493 \pm 0.0010$ & $0.8687 \pm 0.0018$ & $0.9793 \pm 0.0019$ \\
\hline 8883.5713 & $-5.9 \pm 1.6$ & $1 \pm 13$ & $-9.5 \pm 1.7$ & $0.6954 \pm 0.0012$ & $0.5210 \pm 0.0014$ & $0.8485 \pm 0.0009$ & $0.8679 \pm 0.0017$ & $0.9821 \pm 0.0017$ \\
\hline 8884.5713 & $-5.5 \pm 1.2$ & $2.0 \pm 8.8$ & $-4.9 \pm 1.6$ & $0.6912 \pm 0.0011$ & $0.5151 \pm 0.0012$ & $0.8473 \pm 0.0008$ & $0.8675 \pm 0.0014$ & $0.9756 \pm 0.0015$ \\
\hline 8885.5794 & $1.2 \pm 1.7$ & $-8 \pm 13$ & $-5.9 \pm 1.8$ & $0.6859 \pm 0.0013$ & $0.5209 \pm 0.0014$ & $0.8468 \pm 0.0010$ & $0.8701 \pm 0.0017$ & $0.9804 \pm 0.0018$ \\
\hline 8887.5650 & $-9.7 \pm 3.2$ & $17 \pm 27$ & $-9.4 \pm 3.7$ & $0.6919 \pm 0.0027$ & $0.5260 \pm 0.0028$ & $0.8484 \pm 0.0020$ & $0.8684 \pm 0.0035$ & $0.9772 \pm 0.0035$ \\
\hline 8888.7326 & $2.2 \pm 3.7$ & $12 \pm 34$ & $11.0 \pm 4.6$ & $0.6912 \pm 0.0032$ & $0.5210 \pm 0.0034$ & $0.8472 \pm 0.0024$ & $0.8611 \pm 0.0043$ & $0.9758 \pm 0.0041$ \\
\hline 8890.5100 & $-0.1 \pm 2.8$ & $-37 \pm 24$ & $2.3 \pm 2.0$ & $0.6939 \pm 0.0016$ & $0.5295 \pm 0.0018$ & $0.8487 \pm 0.0012$ & $0.8651 \pm 0.0022$ & $0.9798 \pm 0.0022$ \\
\hline 8890.5332 & $-1.5 \pm 1.8$ & $-19 \pm 13$ & $2.2 \pm 1.8$ & $0.6884 \pm 0.0014$ & $0.5268 \pm 0.0016$ & $0.8500 \pm 0.0011$ & $0.8668 \pm 0.0019$ & $0.9816 \pm 0.0020$ \\
\hline 8891.5446 & $-2.6 \pm 1.5$ & $4 \pm 12$ & $6.0 \pm 2.4$ & $0.7054 \pm 0.0012$ & $0.5305 \pm 0.0014$ & $0.8525 \pm 0.0009$ & $0.8677 \pm 0.0016$ & $0.9872 \pm 0.0017$ \\
\hline 8893.5107 & $4.1 \pm 1.6$ & $4 \pm 13$ & $7.9 \pm 2.2$ & $0.6982 \pm 0.0013$ & $0.5270 \pm 0.0014$ & $0.8500 \pm 0.0010$ & $0.8705 \pm 0.0017$ & $0.9800 \pm 0.0018$ \\
\hline 8894.5328 & $-1.0 \pm 1.9$ & $-24 \pm 14$ & $8.1 \pm 1.4$ & $0.6911 \pm 0.0010$ & $0.5297 \pm 0.0011$ & $0.8522 \pm 0.0008$ & $0.8678 \pm 0.0014$ & $0.9802 \pm 0.0015$ \\
\hline 8895.5580 & $0.6 \pm 2.0$ & $-52 \pm 14$ & $-4.9 \pm 2.2$ & $0.6938 \pm 0.0013$ & $0.5265 \pm 0.0014$ & $0.8506 \pm 0.0010$ & $0.8706 \pm 0.0018$ & $0.9865 \pm 0.0018$ \\
\hline 8896.5272 & $3.0 \pm 1.4$ & $-8 \pm 12$ & $0.3 \pm 1.9$ & $0.6989 \pm 0.0011$ & $0.5233 \pm 0.0012$ & $0.8510 \pm 0.0008$ & $0.8682 \pm 0.0015$ & $0.9784 \pm 0.0015$ \\
\hline 8897.5334 & $-3.0 \pm 1.4$ & $-15 \pm 11$ & $1.5 \pm 1.5$ & $0.6990 \pm 0.0012$ & $0.5270 \pm 0.0013$ & $0.8489 \pm 0.0009$ & $0.8642 \pm 0.0016$ & $0.9877 \pm 0.0017$ \\
\hline \multicolumn{9}{|c|}{ HARPS-N } \\
\hline $\begin{array}{l}\text { BJD } \\
(-2450000)\end{array}$ & $\begin{array}{c}\mathrm{RV} \\
\left(\mathrm{m} \mathrm{s}^{-1}\right)\end{array}$ & $\begin{array}{c}\mathrm{CRX} \\
\left(\mathrm{m} \mathrm{s}^{-1} \mathrm{~Np}^{-1}\right)\end{array}$ & $\begin{array}{c}\text { dLW } \\
\left(\mathrm{m}^{2} \mathrm{~s}^{-2}\right)\end{array}$ & $\mathrm{H} \alpha$ & $S_{\text {MWO }}$ & $\log R_{\mathrm{HK}}^{\prime}$ & & \\
\hline 8862.5810 & $6.6 \pm 2.0$ & $11 \pm 17$ & $-12.7 \pm 3.5$ & $0.7144 \pm 0.0026$ & $0.967 \pm 0.043$ & $-4.748 \pm 0.045$ & & \\
\hline 8862.7100 & $2.0 \pm 1.0$ & $-2.2 \pm 7.5$ & $-25.8 \pm 2.0$ & $0.7125 \pm 0.0013$ & $0.991 \pm 0.014$ & $-4.735 \pm 0.038$ & & \\
\hline 8863.6284 & $0.24 \pm 0.60$ & $2.1 \pm 4.8$ & $-30.4 \pm 1.3$ & $0.7154 \pm 0.0010$ & $1.015 \pm 0.008$ & $-4.722 \pm 0.037$ & & \\
\hline 8863.7378 & $-1.32 \pm 0.72$ & $4.7 \pm 5.7$ & $-30.4 \pm 1.3$ & $0.7147 \pm 0.0009$ & $1.005 \pm 0.009$ & $-4.727 \pm 0.037$ & & \\
\hline 8864.6070 & $5.2 \pm 1.0$ & $19.5 \pm 7.6$ & $-28.4 \pm 1.7$ & $0.7144 \pm 0.0013$ & $1.000 \pm 0.012$ & $-4.730 \pm 0.038$ & & \\
\hline 8864.7169 & $5.91 \pm 0.99$ & $7.6 \pm 7.8$ & $-30.4 \pm 1.6$ & $0.7242 \pm 0.0013$ & $1.040 \pm 0.014$ & $-4.709 \pm 0.038$ & & \\
\hline 8865.5889 & $8.28 \pm 0.70$ & $-0.9 \pm 5.7$ & $-29.8 \pm 1.4$ & $0.7156 \pm 0.0012$ & $1.027 \pm 0.010$ & $-4.716 \pm 0.037$ & & \\
\hline 8865.7198 & $4.97 \pm 0.76$ & $8.8 \pm 6.0$ & $-29.0 \pm 1.3$ & $0.7134 \pm 0.0011$ & $1.009 \pm 0.008$ & $-4.725 \pm 0.037$ & & \\
\hline 8869.6175 & $10.75 \pm 0.98$ & $4.5 \pm 7.8$ & $-25.9 \pm 2.2$ & $0.7131 \pm 0.0015$ & $0.986 \pm 0.021$ & $-4.738 \pm 0.039$ & & \\
\hline 8869.7536 & $10.22 \pm 0.89$ & $-4.2 \pm 6.9$ & $-26.1 \pm 1.5$ & $0.7152 \pm 0.0009$ & $1.073 \pm 0.011$ & $-4.692 \pm 0.037$ & & \\
\hline 8870.6093 & $6.0 \pm 1.6$ & $-22 \pm 13$ & $-22.4 \pm 2.4$ & $0.7120 \pm 0.0018$ & $1.031 \pm 0.030$ & $-4.713 \pm 0.040$ & & \\
\hline 8870.6978 & $6.0 \pm 1.6$ & $-21 \pm 13$ & $-22.4 \pm 2.9$ & $0.7087 \pm 0.0020$ & $1.017 \pm 0.033$ & $-4.721 \pm 0.041$ & & \\
\hline 8895.4528 & $11.9 \pm 1.9$ & $7 \pm 15$ & $-27.5 \pm 2.8$ & $0.7159 \pm 0.0024$ & $0.999 \pm 0.042$ & $-4.731 \pm 0.044$ & & \\
\hline 8896.5214 & $11.37 \pm 0.95$ & $-2.2 \pm 7.5$ & $-31.2 \pm 1.9$ & $0.7186 \pm 0.0013$ & $0.985 \pm 0.016$ & $-4.738 \pm 0.038$ & & \\
\hline 8896.6331 & $12.1 \pm 1.2$ & $-2.2 \pm 9.6$ & $-31.7 \pm 1.8$ & $0.7155 \pm 0.0012$ & $1.021 \pm 0.014$ & $-4.719 \pm 0.038$ & & \\
\hline 8897.6418 & $4.6 \pm 1.1$ & $-14.0 \pm 8.4$ & $-34.1 \pm 1.5$ & $0.7166 \pm 0.0012$ & $0.978 \pm 0.012$ & $-4.742 \pm 0.038$ & & \\
\hline 8898.5249 & $6.64 \pm 0.87$ & $-16.4 \pm 6.4$ & $-35.4 \pm 1.3$ & $0.7170 \pm 0.0010$ & $1.009 \pm 0.010$ & $-4.725 \pm 0.037$ & & \\
\hline 8898.6937 & $2.4 \pm 1.0$ & $-8.2 \pm 8.2$ & $-35.0 \pm 1.8$ & $0.7234 \pm 0.0015$ & $0.980 \pm 0.019$ & $-4.741 \pm 0.039$ & & \\
\hline 8905.5116 & $-0.8 \pm 1.4$ & $2 \pm 11$ & $-36.8 \pm 2.6$ & $0.7242 \pm 0.0018$ & $0.989 \pm 0.025$ & $-4.736 \pm 0.040$ & & \\
\hline 8905.6346 & $-0.5 \pm 1.6$ & $-8 \pm 13$ & $-36.3 \pm 2.3$ & $0.7185 \pm 0.0020$ & $0.942 \pm 0.026$ & $-4.763 \pm 0.041$ & & \\
\hline 8925.5936 & $-5.1 \pm 1.5$ & $3 \pm 12$ & $-27.1 \pm 2.3$ & $0.7183 \pm 0.0020$ & $1.033 \pm 0.031$ & $-4.712 \pm 0.040$ & & \\
\hline
\end{tabular}


P. Bluhm et al.: Super-Earth in the radius gap of an M dwarf?

Table A.3. Radial velocity measurements and spectroscopic activity indicators for TOI-1235 from NIR spectra.

\begin{tabular}{|c|c|c|c|}
\hline \multicolumn{4}{|c|}{ CARMENES NIR } \\
\hline $\begin{array}{l}\text { BJD } \\
(-2450000)\end{array}$ & $\begin{array}{c}\mathrm{RV} \\
\left(\mathrm{m} \mathrm{s}^{-1}\right)\end{array}$ & $\begin{array}{c}\mathrm{CRX} \\
\left(\mathrm{m} \mathrm{s}^{-1} \mathrm{~Np}^{-1}\right)\end{array}$ & $\begin{array}{c}\mathrm{dLW} \\
\left(\mathrm{m}^{2} \mathrm{~s}^{-2}\right)\end{array}$ \\
\hline 8796.6534 & $-3.62 \pm 8.7$ & $-15.51 \pm 43$ & $-9.56 \pm 16$ \\
\hline 8807.7242 & $2.7 \pm 11.5$ & $35.98 \pm 51$ & $14.64 \pm 11$ \\
\hline 8815.7113 & $-5.57 \pm 7.0$ & $59.31 \pm 32$ & $20.36 \pm 6.3$ \\
\hline 8816.6574 & $-12.1 \pm 4.3$ & $50.87 \pm 17$ & $26.59 \pm 12$ \\
\hline 8817.7193 & $-28.31 \pm 12.5$ & $91.45 \pm 58$ & $2.89 \pm 24$ \\
\hline 8831.5418 & $14.98 \pm 10.8$ & $25.22 \pm 51$ & $20.13 \pm 16$ \\
\hline 8832.6942 & $-9.92 \pm 9.5$ & $102.59 \pm 39$ & $32.11 \pm 11$ \\
\hline 8846.6695 & $16.88 \pm 14.2$ & $-169.25 \pm 98$ & $-55.07 \pm 11$ \\
\hline 8848.7123 & $9.06 \pm 7.1$ & $21.37 \pm 37$ & $19.54 \pm 5.2$ \\
\hline 8850.6443 & $-7.15 \pm 7.5$ & $41.75 \pm 37$ & $10.7 \pm 6.1$ \\
\hline 8852.6275 & $-3.75 \pm 6.2$ & $-18.49 \pm 31$ & $25.48 \pm 8.5$ \\
\hline 8854.6613 & $3.59 \pm 8.7$ & $-23.5 \pm 40$ & $12.99 \pm 9.2$ \\
\hline 8855.6350 & $-25.78 \pm 14.4$ & $-127.78 \pm 99$ & $-10.78 \pm 13$ \\
\hline 8856.6274 & $-16.5 \pm 5.1$ & $37.01 \pm 23$ & $28.13 \pm 8.2$ \\
\hline 8857.6309 & $0.07 \pm 4.8$ & $12.28 \pm 22$ & $9.51 \pm 7.9$ \\
\hline 8858.6034 & $-12.53 \pm 5.6$ & $-13.31 \pm 23$ & $-8.3 \pm 10$ \\
\hline 8860.6337 & $-7.52 \pm 7.3$ & $95.19 \pm 27$ & $24.34 \pm 11$ \\
\hline 8861.6289 & $-3.69 \pm 5.5$ & $28.43 \pm 26$ & $19.61 \pm 9.8$ \\
\hline 8862.6275 & $10.29 \pm 8.1$ & $84.9 \pm 36$ & $31.28 \pm 9.3$ \\
\hline 8863.6678 & $-27.79 \pm 25.7$ & $56.84 \pm 127$ & $63.0 \pm 29$ \\
\hline 8863.6864 & $-10.75 \pm 13.1$ & $14.07 \pm 65$ & $27.92 \pm 11$ \\
\hline 8864.6146 & $-9.35 \pm 8.6$ & $108.24 \pm 34$ & $44.67 \pm 8.2$ \\
\hline 8865.6222 & $6.09 \pm 9.3$ & $82.43 \pm 42$ & $0.45 \pm 8.3$ \\
\hline 8866.6361 & $-24.99 \pm 19.1$ & $46.87 \pm 108$ & $-107.27 \pm 34$ \\
\hline 8877.5768 & $-11.17 \pm 5.6$ & $7.22 \pm 27$ & $14.44 \pm 6.4$ \\
\hline 8881.5859 & $-7.27 \pm 5.8$ & $61.08 \pm 25$ & $3.89 \pm 6.7$ \\
\hline 8882.5755 & $-5.17 \pm 5.0$ & $-12.44 \pm 24$ & $-2.18 \pm 6.8$ \\
\hline 8883.5726 & $-6.14 \pm 6.6$ & $38.85 \pm 32$ & $-3.2 \pm 6.8$ \\
\hline 8884.5719 & $-7.81 \pm 4.9$ & $24.28 \pm 23$ & $-4.16 \pm 8.1$ \\
\hline 8885.5807 & $-12.04 \pm 7.0$ & $29.82 \pm 34$ & $19.68 \pm 9.0$ \\
\hline 8887.5639 & $-5.08 \pm 10.8$ & $-104.73 \pm 46$ & $-15.27 \pm 25$ \\
\hline 8888.7295 & $-12.98 \pm 17.3$ & $34.81 \pm 85$ & $-27.62 \pm 28$ \\
\hline 8890.5100 & $1.13 \pm 15.4$ & $-5.12 \pm 113$ & $-34.55 \pm 18$ \\
\hline 8890.5349 & $-8.16 \pm 7.1$ & $-20.73 \pm 38$ & $18.13 \pm 10$ \\
\hline 8891.5421 & $-1.74 \pm 4.8$ & $-12.32 \pm 24$ & $10.26 \pm 8.6$ \\
\hline 8893.5073 & $9.0 \pm 7.6$ & $47.15 \pm 45$ & $38.66 \pm 14$ \\
\hline 8894.5324 & $-8.05 \pm 5.3$ & $55.52 \pm 24$ & $5.35 \pm 6.9$ \\
\hline 8895.5593 & $4.62 \pm 6.1$ & $-33.6 \pm 30$ & $3.3 \pm 7.3$ \\
\hline 8896.5263 & $0.14 \pm 5.1$ & $-11.55 \pm 27$ & $32.04 \pm 6.6$ \\
\hline 8897.5325 & $5.19 \pm 13.7$ & $-22.3 \pm 100$ & $-41.89 \pm 19$ \\
\hline 8903.4969 & $-4.6 \pm 10.7$ & $139.99 \pm 63$ & $36.28 \pm 16$ \\
\hline 8904.4842 & $-11.51 \pm 6.1$ & $-19.26 \pm 29$ & $6.46 \pm 10$ \\
\hline
\end{tabular}

\begin{tabular}{lc}
\hline \multicolumn{2}{c}{ iSHELL } \\
$\begin{array}{l}\text { BJD } \\
(-2450000)\end{array}$ & $\begin{array}{c}\text { RV } \\
\left(\mathrm{m} \mathrm{s}^{-1}\right)\end{array}$ \\
\hline 8874.1303 & $-0.71 \pm 9.1$ \\
8875.1161 & $6.61 \pm 11.1$ \\
8895.0886 & $2.6 \pm 4.0$ \\
8899.0817 & $13.07 \pm 5.2$ \\
8901.0644 & $-4.16 \pm 6.1$ \\
\hline
\end{tabular}

